\title{
Exploring 2D Materials Thermodynamic Stability via Machine Learning
}

\author{
Gabriel R. Schleder, ${ }^{*, \dagger, \ddagger}$ Carlos Mera Acosta, ${ }^{\dagger}$ and Adalberto Fazzio*,+, \\ †Center for Natural and Human Sciences, Federal University of $A B C$ (UFABC), \\ 09210-580, Santo André, São Paulo, Brazil \\ †Brazilian Nanotechnology National Laboratory (LNNano)/CNPEM, 13083-970, \\ Campinas, São Paulo,Brazil \\ E-mail: gabriel.schleder@ufabc.edu.br; adalberto.fazzio@lnnano.cnpem.br
}


The final trained XGBoost model uses 91 features in the order: Prototype, six statistical functions (Table 2) for each atomic property of Table $1\left(Z, \epsilon^{h o}, \epsilon^{l u}, \mathcal{I}, \mathcal{E}, r_{s}, r_{p}, r, r_{v}, \chi, \alpha\right.$, $\mathcal{G}, \varnothing$, and $v$ ), and the six SISSO-generated features presented in the manuscript Table 3.

The final model trained parameters are:
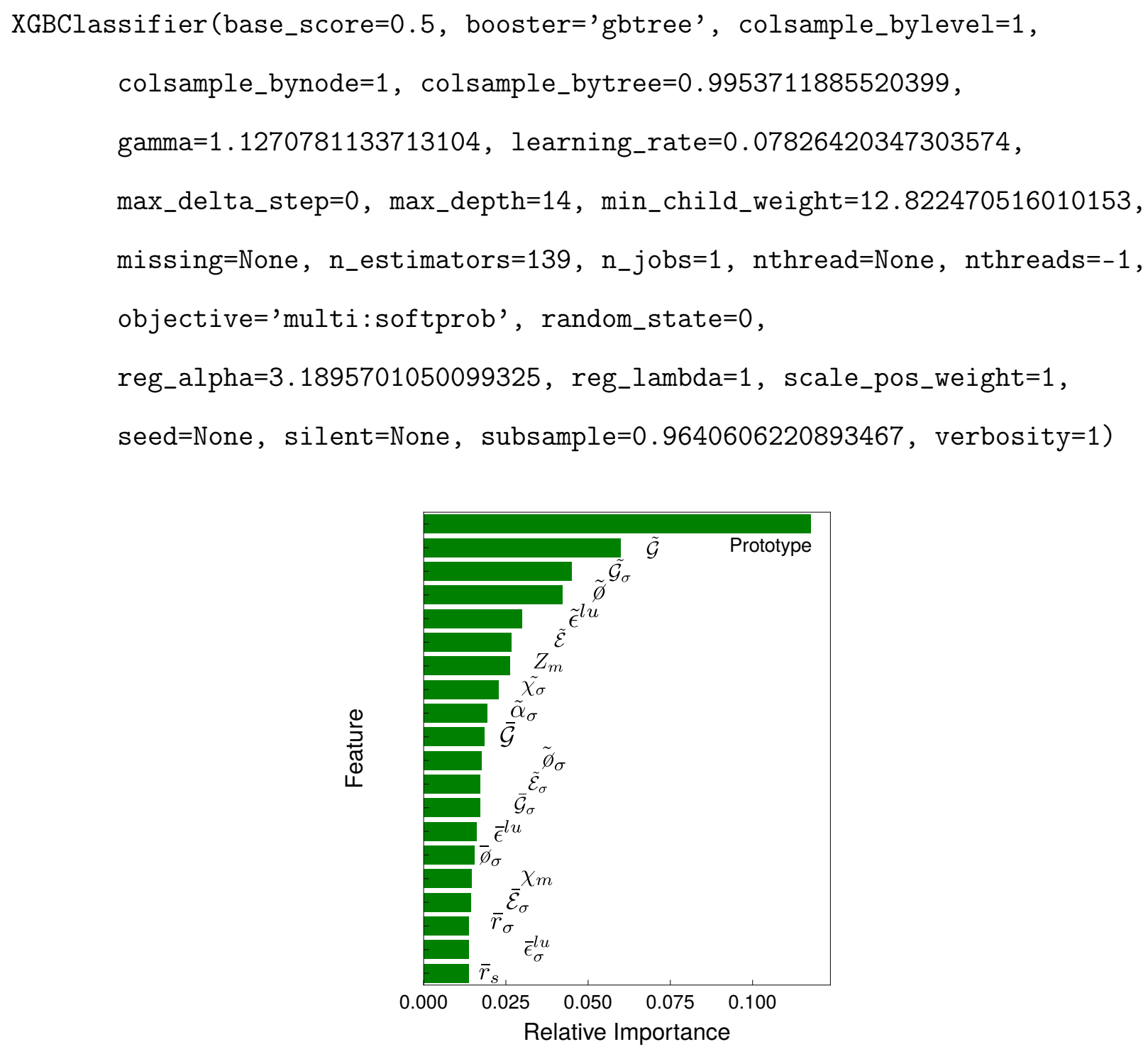

Figure S1: Feature importance for the classification model without the SISSO-generated features. 
The next figures show the SISSO correlation scatter plots of formation energies comparing the DFT calculated values with the SISSO machine learning regression models for each 2D materials stability class.
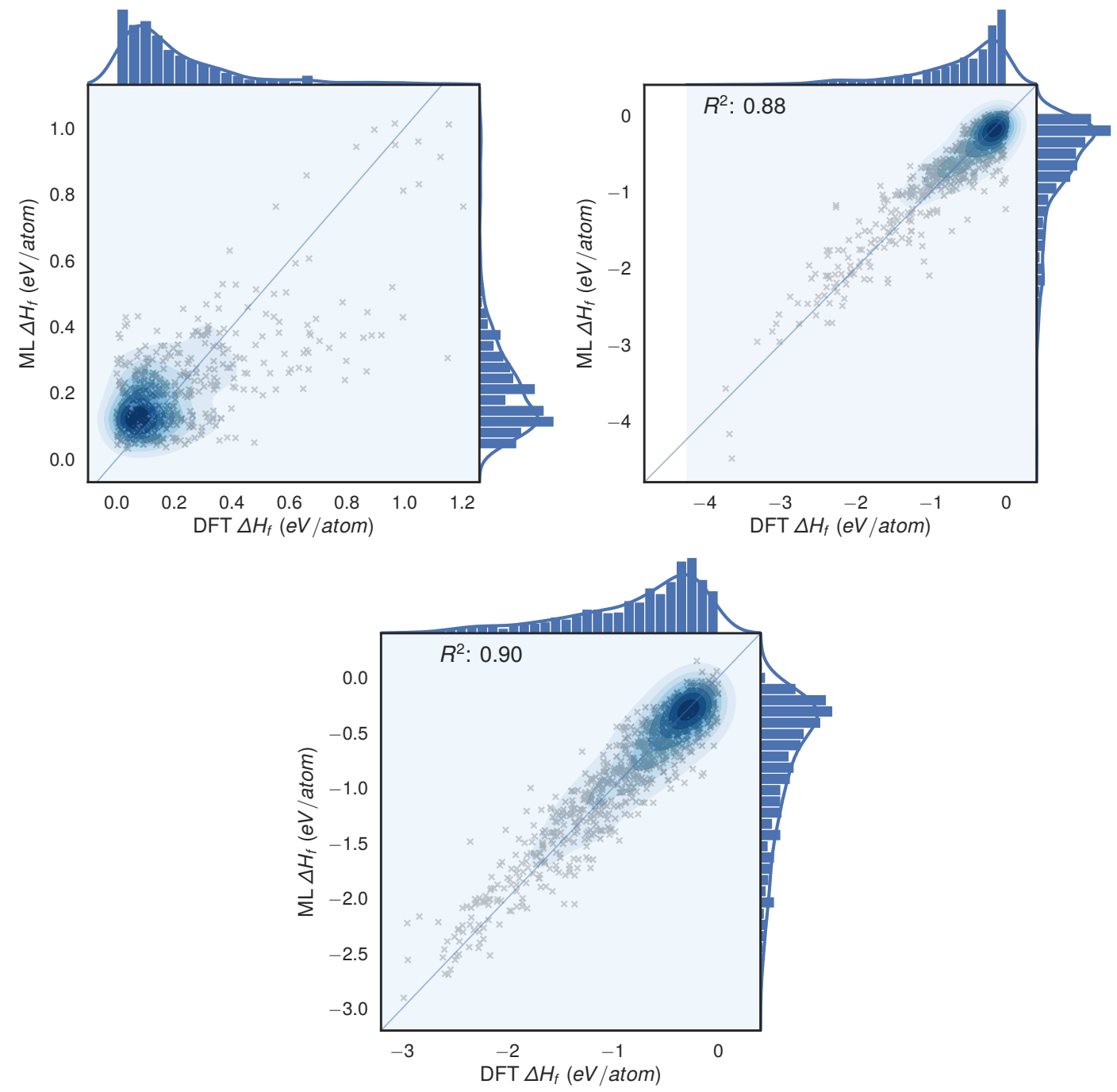

Figure S2: Correlation scatter plot of formation energies comparing the DFT calculated values with the regression model obtained via SISSO machine learning for the classes low, medium, and high stability. 
Table S1: SISSO machine learning linear regression coefficients $c$ for the three materials classes.

\begin{tabular}{cllll} 
& $c_{1}$ & $c_{2}$ & $c_{3}$ & $c_{4}$ \\
\hline Low & -2.5163 & $0.40869 \mathrm{E}-05$ & 0.12500 & 0.019152 \\
Medium & -0.51251 & -0.026682 & 0.0035589 & 0.032611 \\
High & -1.4566 & -0.30172 & 0.18729 & -0.18979
\end{tabular}

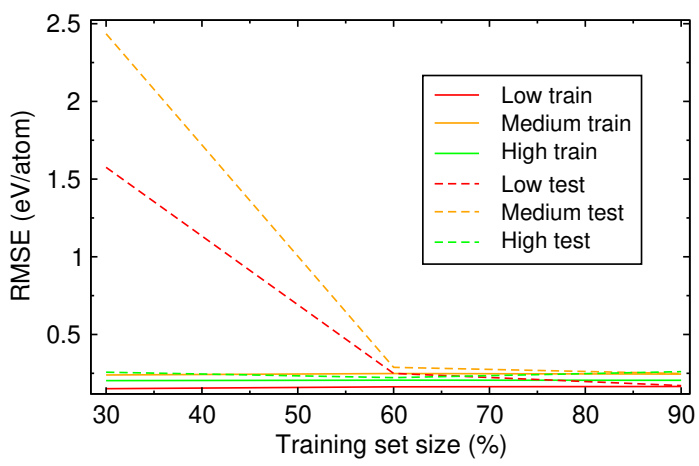

Figure S3: Learning curve of the regression models. Training error is obtained with 5-fold cross-validation. 
Table S2: Screened materials for water splitting. The formula, prototype, space group, band gap, and band edges (valence band maximum and conduction band mininmum) are presented.

\begin{tabular}{|c|c|c|c|c|c|c|}
\hline Formula & Prototype & Space group & $\begin{array}{c}\text { Band gap }\left(\mathrm{G}_{0} \mathrm{~W}_{0}\right) \\
(\mathrm{eV})\end{array}$ & $\begin{array}{l}\text { VBM } \\
(\mathrm{eV})\end{array}$ & $\begin{array}{l}\text { CBM } \\
(\mathrm{eV})\end{array}$ & Direct gap \\
\hline $\mathrm{CrO} 2$ & MoS2 & P-6m2 & 1.230 & -7.823 & -6.593 & False \\
\hline PtTe2 & $\mathrm{CdI} 2$ & P-3m1 & 1.242 & -4.897 & -3.655 & False \\
\hline FeI2 & $\mathrm{CdI} 2$ & $\mathrm{P}-3 \mathrm{~m} 1$ & 1.256 & -4.585 & -3.329 & False \\
\hline CrSSe & MoSSe & P3m1 & 1.294 & -5.793 & -4.499 & True \\
\hline TiSSe & MoSSe & P3m1 & 1.333 & -6.422 & -5.089 & False \\
\hline $\operatorname{PdBr} 2$ & $\mathrm{CdI} 2$ & $\mathrm{P}-3 \mathrm{~m} 1$ & 1.334 & -6.104 & -4.770 & False \\
\hline YBr2 & MoS2 & $\mathrm{P}-6 \mathrm{~m} 2$ & 1.353 & -4.481 & -3.128 & False \\
\hline WTe2 & MoS2 & $\mathrm{P}-6 \mathrm{~m} 2$ & 1.377 & -4.769 & -3.393 & True \\
\hline TiClI & MoSSe & P3m1 & 1.383 & -3.936 & -2.553 & False \\
\hline BiITe & BiTeI & P3m1 & 1.419 & -6.443 & -5.024 & False \\
\hline HfI2 & MoS2 & P-6m2 & 1.421 & -3.621 & -2.200 & False \\
\hline TiBr2 & MoS2 & P-6m2 & 1.421 & -3.802 & -2.380 & False \\
\hline $\mathrm{ZrI} 2$ & MoS2 & $\mathrm{P}-6 \mathrm{~m} 2$ & 1.428 & -3.708 & -2.280 & False \\
\hline $\mathrm{CrS} 2$ & MoS2 & $\mathrm{P}-6 \mathrm{~m} 2$ & 1.448 & -6.167 & -4.719 & True \\
\hline $\mathrm{TiBrCl}$ & MoSSe & P3m1 & 1.473 & -4.088 & -2.616 & False \\
\hline TiSe2 & MoS2 & $\mathrm{P}-6 \mathrm{~m} 2$ & 1.481 & -6.417 & -4.936 & False \\
\hline MoTe2 & MoS2 & $\mathrm{P}-6 \mathrm{~m} 2$ & 1.563 & -4.719 & -3.156 & True \\
\hline $\mathrm{ZrBrI}$ & MoSSe & P3m1 & 1.590 & -3.884 & -2.294 & False \\
\hline $\mathrm{YCl} 2$ & MoS2 & $\mathrm{P}-6 \mathrm{~m} 2$ & 1.611 & -4.715 & -3.104 & False \\
\hline HfBrI & MoSSe & P3m1 & 1.612 & -3.820 & -2.209 & False \\
\hline $\mathrm{PdSe} 2$ & $\mathrm{CdI} 2$ & $\mathrm{P}-3 \mathrm{~m} 1$ & 1.615 & -6.000 & -4.385 & False \\
\hline $\mathrm{Pb} 2 \mathrm{Se} 2$ & $\mathrm{PbS}$ & $\mathrm{P} 1$ & 1.623 & -5.261 & -3.639 & False \\
\hline
\end{tabular}




$\begin{array}{lcccccc}\text { TiCl2 } & \text { MoS2 } & \text { P-6m2 } & 1.636 & -4.250 & -2.614 & \text { False } \\ \text { ISbTe } & \text { BiTeI } & \text { P3m1 } & 1.676 & -6.073 & -4.396 & \text { False } \\ \text { BiBrTe } & \text { BiTeI } & \text { P3m1 } & 1.690 & -6.836 & -5.145 & \text { False } \\ \text { ZrSe2 } & \text { CdI2 } & \text { P-3m1 } & 1.691 & -6.311 & -4.620 & \text { False } \\ \text { FeCl2 } & \text { CdI2 } & \text { P-3m1 } & 1.721 & -5.232 & -3.511 & \text { False } \\ \text { BiClTe } & \text { BiTeI } & \text { P3m1 } & 1.736 & -6.888 & -5.151 & \text { True } \\ \text { ZrBr2 } & \text { MoS2 } & \text { P-6m2 } & 1.737 & -4.043 & -2.306 & \text { False } \\ \text { ZrClI } & \text { MoSSe } & \text { P3m1 } & 1.744 & -4.073 & -2.329 & \text { False } \\ \text { HfBr2 } & \text { MoS2 } & \text { P-6m2 } & 1.759 & -3.984 & -2.225 & \text { False } \\ \text { BiISe } & \text { BiTeI } & \text { P3m1 } & 1.772 & -6.887 & -5.115 & \text { False } \\ \text { HfClI } & \text { MoSSe } & \text { P3m1 } & 1.783 & -4.029 & -2.246 & \text { False } \\ \text { HfSe2 } & \text { CdI2 } & \text { P-3m1 } & 1.788 & -6.325 & -4.538 & \text { False } \\ \text { WSeTe } & \text { MoSSe } & \text { P3m1 } & 1.800 & -5.085 & -3.285 & \text { True } \\ \text { NiS2 } & \text { CdI2 } & \text { P-3m1 } & 1.868 & -6.523 & -4.655 & \text { False } \\ \text { ZrBrCl } & \text { MoSSe } & \text { P3m1 } & 1.878 & -4.232 & -2.354 & \text { False } \\ \text { SnSe2 } & \text { CdI2 } & \text { P-3m1 } & 1.912 & -6.884 & -4.972 & \text { False } \\ \text { HfBrCl } & \text { MoSSe } & \text { P3m1 } & 1.926 & -4.179 & -2.253 & \text { False } \\ \text { RuI2 } & \text { CdI2 } & \text { P-3m1 } & 1.988 & -4.621 & -2.633 & \text { True } \\ \text { ZrCl2 } & \text { MoS2 } & \text { P-6m2 } & 1.993 & -4.430 & -2.438 & \text { False } \\ \text { ISbSe } & \text { BiTeI } & \text { P3m1 } & 1.994 & -6.431 & -4.438 & \text { False } \\ \text { P4 } & \text { P } & \text { Pma2 } & 2.025 & -5.956 & -3.931 & \text { False } \\ \text { AsISe } & \text { BiTeI } & \text { P3m1 } & 2.030 & -6.311 & -4.281 & \text { False } \\ \text { BiIS } & \text { BiTeI } & \text { P3m1 } & 2.042 & -7.188 & -5.146 & \text { False } \\ \text { PbTe } & \text { GeSe } & \text { P3m1 } & 2.059 & -5.519 & -3.460 & \text { True } \\ \text { WrTe } & \text { MoSSe } & \text { P3m1 } & 2.065 & -5.502 & -3.437 & \text { False } \\ \text { BrSbTe } & \text { BiTeI } & \text { P3m1 } & 2.070 & -6.605 & -4.535 & \text { False } \\ \text { MoS2 } & \text { P-6m2 } & 2.092 & -4.373 & -2.282 & \text { False }\end{array}$




$\begin{array}{lcccccc}\text { WSe2 } & \text { MoS2 } & \text { P-6m2 } & 2.104 & -5.316 & -3.212 & \text { True } \\ \text { MoSe2 } & \text { MoS2 } & \text { P-6m2 } & 2.119 & -5.270 & -3.151 & \text { True } \\ \text { BrSbSe } & \text { BiTeI } & \text { P3m1 } & 2.164 & -6.898 & -4.734 & \text { False } \\ \text { BiClSe } & \text { BiTeI } & \text { P3m1 } & 2.202 & -7.580 & -5.377 & \text { False } \\ \text { AsBrTe } & \text { BiTeI } & \text { P3m1 } & 2.227 & -6.582 & -4.355 & \text { False } \\ \text { ScSe2 } & \text { MoS2 } & \text { P-6m2 } & 2.229 & -6.803 & -4.574 & \text { False } \\ \text { ISSb } & \text { BiTeI } & \text { P3m1 } & 2.260 & -6.667 & -4.407 & \text { False } \\ \text { GeS2 } & \text { CdI2 } & \text { P-3m1 } & 2.266 & -7.532 & -5.266 & \text { False } \\ \text { AsBrSe } & \text { BiTeI } & \text { P3m1 } & 2.274 & -6.803 & -4.529 & \text { False } \\ \text { WSSe } & \text { MoSSe } & \text { P3m1 } & 2.314 & -5.689 & -3.375 & \text { True } \\ \text { MoO2 } & \text { MoS2 } & \text { P-6m2 } & 2.317 & -6.912 & -4.596 & \text { False } \\ \text { MoSSe } & \text { MoSSe } & \text { P3m1 } & 2.328 & -5.620 & -3.292 & \text { True } \\ \text { BiBrS } & \text { BiTeI } & \text { P3m1 } & 2.335 & -7.679 & -5.344 & \text { False } \\ \text { NiO2 } & \text { CdI2 } & \text { P-3m1 } & 2.346 & -8.200 & -5.853 & \text { False } \\ \text { AsIS } & \text { BiTeI } & \text { P3m1 } & 2.356 & -6.614 & -4.258 & \text { False } \\ \text { PtSe2 } & \text { CdI2 } & \text { P-3m1 } & 2.381 & -6.245 & -3.864 & \text { False } \\ \text { ClSbTe } & \text { BiTeI } & \text { P3m1 } & 2.382 & -6.930 & -4.548 & \text { False } \\ \text { RuBr2 } & \text { CdI2 } & \text { P-3m1 } & 2.403 & -4.878 & -2.475 & \text { False } \\ \text { PdCl2 } & \text { CdI2 } & \text { P-3m1 } & 2.407 & -7.041 & -4.635 & \text { False } \\ \text { BrSSb } & \text { BiTeI } & \text { P3m1 } & 2.419 & -7.167 & -4.748 & \text { False } \\ \text { PdS2 } & \text { CdI2 } & \text { P-3m1 } & 2.441 & -7.043 & -4.602 & \text { False } \\ \text { AsBrS } & \text { BiTeI } & \text { P3m1 } & 2.514 & -7.058 & -4.544 & \text { False } \\ \text { WS2 } & \text { MoS2 } & \text { P-6m2 } & 2.532 & -6.018 & -3.486 & \text { True } \\ \text { MoS2 } & \text { MoS2 } & \text { P-6m2 } & 2.533 & -5.961 & -3.428 & \text { True } \\ \text { AsClSe } & \text { BiTeI } & \text { P3m1 } & 2.561 & -7.192 & -4.632 & \text { False } \\ \text { NiBr2 } & \text { CdI2 } & \text { P-3m1 } & 2.616 & -6.660 & -4.044 & \text { False } \\ \text { GeTe } & \text { GeSe } & \text { P3m1 } & 2.647 & -5.812 & -3.165 & \text { False }\end{array}$




$\begin{array}{lllllll}\text { WO2 } & \text { MoS2 } & \text { P-6m2 } & 2.706 & -7.004 & -4.297 & \text { False } \\ \text { ZrS2 } & \text { CdI2 } & \text { P-3m1 } & 2.885 & -7.501 & -4.616 & \text { False } \\ \text { PdO2 } & \text { CdI2 } & \text { P-3m1 } & 2.903 & -7.987 & -5.084 & \text { False } \\ \text { HfS2 } & \text { CdI2 } & \text { P-3m1 } & 2.938 & -7.505 & -4.567 & \text { False } \\ \text { PtS2 } & \text { CdI2 } & \text { P-3m1 } & 2.945 & -6.969 & -4.024 & \text { False } \\ \text { PbO2 } & \text { CdI2 } & \text { P-3m1 } & 2.995 & -9.240 & -6.246 & \text { False } \\ \text { VBr2 } & \text { CdI2 } & \text { P-3m1 } & 3.013 & -4.855 & -1.842 & \text { False } \\ \text { Al2Te2 } & \text { GaS } & \text { P-6m2 } & 3.016 & -6.289 & -3.273 & \text { False } \\ \text { In2S2 } & \text { GaS } & \text { P-6m2 } & 3.149 & -7.530 & -4.381 & \text { False } \\ \text { SnS2 } & \text { CdI2 } & \text { P-3m1 } & 3.150 & -7.942 & -4.792 & \text { False } \\ \text { GeI2 } & \text { CdI2 } & \text { P-3m1 } & 3.348 & -6.846 & -3.498 & \text { False } \\ \text { NiCl2 } & \text { CdI2 } & \text { P-3m1 } & 3.424 & -7.076 & -3.652 & \text { False } \\ \text { Ga2Se2 } & \text { GaS } & \text { P-6m2 } & 3.445 & -6.871 & -3.426 & \text { False } \\ \text { VCl2 } & \text { CdI2 } & \text { P-3m1 } & 3.456 & -5.203 & -1.747 & \text { False }\end{array}$


Table S3: Thermodynamic stability prediction for compounds III-V-VI $\mathrm{VI}_{4}$ and III-V-VI ${ }_{2}$ in different structural prototypes.

\begin{tabular}{|c|c|c|}
\hline Formula & Prototype & Prediction \\
\hline $\mathrm{BNO}_{4}$ & MoS2 & MEDIUM \\
\hline $\mathrm{BNS}_{4}$ & MoS2 & MEDIUM \\
\hline $\mathrm{BNSe}_{4}$ & MoS2 & LOW \\
\hline $\mathrm{BNTe}_{4}$ & MoS2 & MEDIUM \\
\hline $\mathrm{BPO}_{4}$ & MoS2 & MEDIUM \\
\hline $\mathrm{BPS}_{4}$ & MoS2 & LOW \\
\hline $\mathrm{BPSe}_{4}$ & MoS2 & LOW \\
\hline $\mathrm{BPTe}_{4}$ & MoS2 & LOW \\
\hline $\mathrm{BAsO}_{4}$ & MoS2 & MEDIUM \\
\hline $\mathrm{BAsS}_{4}$ & MoS2 & LOW \\
\hline $\mathrm{BAsSe}_{4}$ & MoS2 & LOW \\
\hline $\mathrm{BAsTe}_{4}$ & MoS2 & LOW \\
\hline $\mathrm{BSbO}_{4}$ & MoS2 & MEDIUM \\
\hline $\mathrm{BSbS}_{4}$ & MoS2 & HIGH \\
\hline $\mathrm{BSbSe}_{4}$ & MoS2 & LOW \\
\hline $\mathrm{BSbTe}_{4}$ & MoS2 & LOW \\
\hline $\mathrm{BBiO}_{4}$ & MoS2 & MEDIUM \\
\hline $\mathrm{BBiS}_{4}$ & MoS2 & LOW \\
\hline $\mathrm{BBiSe}_{4}$ & MoS2 & LOW \\
\hline $\mathrm{BBiTe}_{4}$ & MoS2 & LOW \\
\hline $\mathrm{AlNO}_{4}$ & MoS2 & MEDIUM \\
\hline $\mathrm{AlNS}_{4}$ & MoS2 & MEDIUM \\
\hline $\mathrm{AlNSe}_{4}$ & MoS2 & MEDIUM \\
\hline $\mathrm{AlNTe}_{4}$ & MoS2 & LOW \\
\hline
\end{tabular}




\begin{tabular}{|c|c|c|}
\hline $\mathrm{AlPO}_{4}$ & MoS2 & MEDIUM \\
\hline $\mathrm{AlPS}_{4}$ & MoS2 & HIGH \\
\hline $\mathrm{AlPSe}_{4}$ & MoS2 & HIGH \\
\hline $\mathrm{AlPTe}_{4}$ & MoS2 & HIGH \\
\hline $\mathrm{AlAsO}_{4}$ & MoS2 & MEDIUM \\
\hline $\mathrm{AlAsS}_{4}$ & MoS2 & HIGH \\
\hline $\mathrm{AlAsSe}_{4}$ & MoS2 & HIGH \\
\hline $\mathrm{AlAsTe}_{4}$ & MoS2 & LOW \\
\hline $\mathrm{AlSbO}_{4}$ & MoS2 & MEDIUM \\
\hline $\mathrm{AlSbS}_{4}$ & MoS2 & HIGH \\
\hline $\mathrm{AlSbSe}_{4}$ & MoS2 & HIGH \\
\hline $\mathrm{AlSbTe}_{4}$ & MoS2 & HIGH \\
\hline $\mathrm{AlBiO}_{4}$ & MoS2 & MEDIUM \\
\hline $\mathrm{AlBiS}_{4}$ & MoS2 & HIGH \\
\hline $\mathrm{AlBiSe}_{4}$ & MoS2 & HIGH \\
\hline $\mathrm{AlBiTe}_{4}$ & MoS2 & HIGH \\
\hline $\mathrm{GaNO}_{4}$ & MoS2 & MEDIUM \\
\hline $\mathrm{GaNS}_{4}$ & MoS2 & MEDIUM \\
\hline $\mathrm{GaNSe}_{4}$ & MoS2 & LOW \\
\hline $\mathrm{GaNTe}_{4}$ & MoS2 & LOW \\
\hline $\mathrm{GaPO}_{4}$ & MoS2 & MEDIUM \\
\hline $\mathrm{GaPS}_{4}$ & MoS2 & HIGH \\
\hline $\mathrm{GaPSe}_{4}$ & MoS2 & HIGH \\
\hline $\mathrm{GaPTe}_{4}$ & MoS2 & LOW \\
\hline $\mathrm{GaAsO}_{4}$ & MoS2 & MEDIUM \\
\hline $\mathrm{GaAsS}_{4}$ & MoS2 & HIGH \\
\hline $\mathrm{GaAsSe}_{4}$ & MoS2 & LOW \\
\hline
\end{tabular}




\begin{tabular}{|c|c|c|}
\hline $\mathrm{GaAsTe}_{4}$ & MoS2 & LOW \\
\hline $\mathrm{GaSbO}_{4}$ & MoS2 & MEDIUM \\
\hline $\mathrm{GaSbS}_{4}$ & MoS2 & $\mathrm{HIGH}$ \\
\hline $\mathrm{GaSbSe}_{4}$ & MoS2 & $\mathrm{HIGH}$ \\
\hline $\mathrm{GaSbTe}_{4}$ & MoS2 & LOW \\
\hline $\mathrm{GaBiO}_{4}$ & MoS2 & MEDIUM \\
\hline $\mathrm{GaBiS}_{4}$ & MoS2 & HIGH \\
\hline $\mathrm{GaBiSe}_{4}$ & MoS2 & $\mathrm{HIGH}$ \\
\hline $\mathrm{GaBiTe}_{4}$ & MoS2 & HIGH \\
\hline $\mathrm{InNO}_{4}$ & MoS2 & MEDIUM \\
\hline $\mathrm{InNS}_{4}$ & MoS2 & LOW \\
\hline $\mathrm{InNSe}_{4}$ & MoS2 & LOW \\
\hline $\mathrm{InNTe}_{4}$ & MoS2 & LOW \\
\hline $\mathrm{InPO}_{4}$ & MoS2 & MEDIUM \\
\hline $\mathrm{InPS}_{4}$ & MoS2 & HIGH \\
\hline $\mathrm{InPSe}_{4}$ & MoS2 & HIGH \\
\hline $\mathrm{InPTe}_{4}$ & MoS2 & HIGH \\
\hline $\mathrm{InAsO}_{4}$ & MoS2 & MEDIUM \\
\hline $\operatorname{InAsS}_{4}$ & MoS2 & HIGH \\
\hline $\mathrm{InAsSe}_{4}$ & MoS2 & $\mathrm{HIGH}$ \\
\hline $\mathrm{InAsTe}_{4}$ & MoS2 & LOW \\
\hline $\mathrm{InSbO}_{4}$ & MoS2 & MEDIUM \\
\hline $\mathrm{InSbS}_{4}$ & MoS2 & HIGH \\
\hline $\mathrm{InSbSe}_{4}$ & MoS2 & HIGH \\
\hline $\mathrm{InSbTe}_{4}$ & MoS2 & HIGH \\
\hline $\mathrm{InBiO}_{4}$ & MoS2 & MEDIUM \\
\hline $\mathrm{InBiS}_{4}$ & MoS2 & HIGH \\
\hline
\end{tabular}




$\begin{array}{lcc}\text { InBiSe }_{4} & \text { MoS2 } & \text { HIGH } \\ \text { InBiTe }_{4} & \text { MoS2 } & \text { HIGH } \\ \text { TlNO }_{4} & \text { MoS2 } & \text { MEDIUM } \\ \text { TlNS }_{4} & \text { MoS2 } & \text { LOW } \\ \text { TlNSe }_{4} & \text { MoS2 } & \text { LOW } \\ \text { TlNTe }_{4} & \text { MoS2 } & \text { LOW } \\ \text { TlPO }_{4} & \text { MoS2 } & \text { HIGH } \\ \text { TlPS }_{4} & \text { MoS2 } & \text { HIGH } \\ \text { TlPSe }_{4} & \text { MoS2 } & \text { HIGH } \\ \text { TlPTe }_{4} & \text { MoS2 } & \text { HIGH } \\ \text { TlAsO }_{4} & \text { MoS2 } & \text { HIGH } \\ \text { TlAsS }_{4} & \text { MoS2 } & \text { HIGH } \\ \text { TlAsSe }_{4} & \text { MoS2 } & \text { HIGH } \\ \text { TlAsTe }_{4} & \text { MoS2 } & \text { LOW } \\ \text { TlSbO }_{4} & \text { MoS2 } & \text { HIGH } \\ \text { TlSbS }_{4} & \text { MoS2 } & \text { HIGH } \\ \text { TlSbSe }_{4} & \text { MoS2 } & \text { HIGH } \\ \text { TlSbTe }_{4} & \text { MoS2 } & \text { HIGH } \\ \text { TlBiO }_{4} & \text { MoS2 } & \text { HIGH } \\ \text { TlBiS }_{4} & \text { MoS2 } & \text { HIGH } \\ \text { TlBiSe }_{4} & \text { MoS2 } & \text { HIGH } \\ \text { TlBiTe }_{4} & \text { MoS2 } & \text { HIGH } \\ \text { BNO }_{4} & \text { CdI2 } & \text { MEDIUM } \\ \text { BNS }_{4} & \text { CdI2 } & \text { LOW } \\ \text { BNSe }_{4} & \text { CdI2 } & \text { LOW } \\ \text { BNTe }_{4} & \text { CdI2 } & \text { LOW } \\ & \text { CdI2 } & \text { MEDIUM }\end{array}$




\begin{tabular}{|c|c|c|}
\hline $\mathrm{BPS}_{4}$ & CdI2 & LOW \\
\hline $\mathrm{BPSe}_{4}$ & $\mathrm{CdI} 2$ & LOW \\
\hline $\mathrm{BPTe}_{4}$ & CdI2 & LOW \\
\hline $\mathrm{BAsO}_{4}$ & $\mathrm{CdI} 2$ & MEDIUM \\
\hline $\mathrm{BAsS}_{4}$ & $\mathrm{CdI} 2$ & LOW \\
\hline $\mathrm{BAsSe}_{4}$ & $\mathrm{CdI} 2$ & LOW \\
\hline $\mathrm{BAsTe}_{4}$ & $\mathrm{CdI} 2$ & LOW \\
\hline $\mathrm{BSbO}_{4}$ & $\mathrm{CdI} 2$ & MEDIUM \\
\hline $\mathrm{BSbS}_{4}$ & CdI2 & HIGH \\
\hline $\mathrm{BSbSe}_{4}$ & CdI2 & LOW \\
\hline $\mathrm{BSbTe}_{4}$ & $\mathrm{CdI} 2$ & LOW \\
\hline $\mathrm{BBiO}_{4}$ & $\mathrm{CdI} 2$ & MEDIUM \\
\hline $\mathrm{BBiS}_{4}$ & CdI2 & LOW \\
\hline $\mathrm{BBiSe}_{4}$ & $\mathrm{CdI} 2$ & LOW \\
\hline $\mathrm{BBiTe}_{4}$ & $\mathrm{CdI} 2$ & LOW \\
\hline $\mathrm{AlNO}_{4}$ & CdI2 & MEDIUM \\
\hline $\mathrm{AlNS}_{4}$ & CdI2 & MEDIUM \\
\hline $\mathrm{AlNSe}_{4}$ & CdI2 & MEDIUM \\
\hline $\mathrm{AlNTe}_{4}$ & CdI2 & LOW \\
\hline $\mathrm{AlPO}_{4}$ & $\mathrm{CdI} 2$ & HIGH \\
\hline $\mathrm{AlPS}_{4}$ & CdI2 & HIGH \\
\hline $\mathrm{AlPSe}_{4}$ & CdI2 & HIGH \\
\hline $\mathrm{AlPTe}_{4}$ & CdI2 & HIGH \\
\hline $\mathrm{AlAsO}_{4}$ & CdI2 & HIGH \\
\hline $\mathrm{AlAsS}_{4}$ & $\mathrm{CdI} 2$ & HIGH \\
\hline $\mathrm{AlAsSe}_{4}$ & CdI2 & HIGH \\
\hline $\mathrm{AlAsTe}_{4}$ & $\mathrm{CdI} 2$ & HIGH \\
\hline
\end{tabular}




\begin{tabular}{|c|c|c|}
\hline $\mathrm{AlSbO}_{4}$ & $\mathrm{CdI} 2$ & HIGH \\
\hline $\mathrm{AlSbS}_{4}$ & $\mathrm{CdI} 2$ & HIGH \\
\hline $\mathrm{AlSbSe}_{4}$ & $\mathrm{CdI} 2$ & HIGH \\
\hline $\mathrm{AlSbTe}_{4}$ & $\mathrm{CdI} 2$ & HIGH \\
\hline $\mathrm{AlBiO}_{4}$ & $\mathrm{CdI} 2$ & HIGH \\
\hline $\mathrm{AlBiS}_{4}$ & $\mathrm{CdI} 2$ & HIGH \\
\hline $\mathrm{AlBiSe}_{4}$ & $\mathrm{CdI} 2$ & HIGH \\
\hline $\mathrm{AlBiTe}_{4}$ & $\mathrm{CdI} 2$ & HIGH \\
\hline $\mathrm{GaNO}_{4}$ & $\mathrm{CdI} 2$ & HIGH \\
\hline $\mathrm{GaNS}_{4}$ & $\mathrm{CdI} 2$ & MEDIUM \\
\hline $\mathrm{GaNSe}_{4}$ & $\mathrm{CdI} 2$ & LOW \\
\hline $\mathrm{GaNTe}_{4}$ & $\mathrm{CdI} 2$ & LOW \\
\hline $\mathrm{GaPO}_{4}$ & $\mathrm{CdI} 2$ & HIGH \\
\hline $\mathrm{GaPS}_{4}$ & $\mathrm{CdI} 2$ & HIGH \\
\hline $\mathrm{GaPSe}_{4}$ & $\mathrm{CdI} 2$ & HIGH \\
\hline $\mathrm{GaPTe}_{4}$ & $\mathrm{CdI} 2$ & HIGH \\
\hline $\mathrm{GaAsO}_{4}$ & $\mathrm{CdI} 2$ & HIGH \\
\hline $\mathrm{GaAsS}_{4}$ & $\mathrm{CdI} 2$ & HIGH \\
\hline $\mathrm{GaAsSe}_{4}$ & $\mathrm{CdI} 2$ & HIGH \\
\hline $\mathrm{GaAsTe}_{4}$ & $\mathrm{CdI} 2$ & HIGH \\
\hline $\mathrm{GaSbO}_{4}$ & $\mathrm{CdI} 2$ & HIGH \\
\hline $\mathrm{GaSbS}_{4}$ & $\mathrm{CdI} 2$ & HIGH \\
\hline $\mathrm{GaSbSe}_{4}$ & $\mathrm{CdI} 2$ & HIGH \\
\hline $\mathrm{GaSbTe}_{4}$ & $\mathrm{CdI} 2$ & HIGH \\
\hline $\mathrm{GaBiO}_{4}$ & $\mathrm{CdI} 2$ & $\mathrm{HIGH}$ \\
\hline $\mathrm{GaBiS}_{4}$ & $\mathrm{CdI} 2$ & HIGH \\
\hline $\mathrm{GaBiSe}_{4}$ & $\mathrm{CdI} 2$ & $\mathrm{HIGH}$ \\
\hline
\end{tabular}




\begin{tabular}{|c|c|c|}
\hline $\mathrm{GaBiTe}_{4}$ & $\mathrm{CdI} 2$ & $\mathrm{HIGH}$ \\
\hline $\mathrm{InNO}_{4}$ & $\mathrm{CdI} 2$ & $\mathrm{HIGH}$ \\
\hline $\mathrm{InNS}_{4}$ & $\mathrm{CdI} 2$ & LOW \\
\hline $\mathrm{InNSe}_{4}$ & $\mathrm{CdI} 2$ & LOW \\
\hline $\mathrm{InNTe}_{4}$ & CdI2 & LOW \\
\hline $\mathrm{InPO}_{4}$ & $\mathrm{CdI} 2$ & $\mathrm{HIGH}$ \\
\hline $\mathrm{InPS}_{4}$ & $\mathrm{CdI} 2$ & $\mathrm{HIGH}$ \\
\hline $\mathrm{InPSe}_{4}$ & $\mathrm{CdI} 2$ & $\mathrm{HIGH}$ \\
\hline $\mathrm{InPTe}_{4}$ & CdI2 & $\mathrm{HIGH}$ \\
\hline $\mathrm{InAsO}_{4}$ & $\mathrm{CdI} 2$ & $\mathrm{HIGH}$ \\
\hline $\mathrm{InAsS}_{4}$ & $\mathrm{CdI} 2$ & $\mathrm{HIGH}$ \\
\hline $\mathrm{InAsSe}_{4}$ & CdI2 & $\mathrm{HIGH}$ \\
\hline $\mathrm{InAsTe}_{4}$ & CdI2 & $\mathrm{HIGH}$ \\
\hline $\mathrm{InSbO}_{4}$ & $\mathrm{CdI} 2$ & $\mathrm{HIGH}$ \\
\hline $\mathrm{InSbS}_{4}$ & CdI2 & HIGH \\
\hline $\mathrm{InSbSe}_{4}$ & CdI2 & $\mathrm{HIGH}$ \\
\hline $\mathrm{InSbTe}_{4}$ & $\mathrm{CdI} 2$ & $\mathrm{HIGH}$ \\
\hline $\mathrm{InBiO}_{4}$ & CdI2 & $\mathrm{HIGH}$ \\
\hline $\mathrm{InBiS}_{4}$ & CdI2 & $\mathrm{HIGH}$ \\
\hline $\mathrm{InBiSe}_{4}$ & CdI2 & $\mathrm{HIGH}$ \\
\hline $\mathrm{InBiTe}_{4}$ & $\mathrm{CdI} 2$ & $\mathrm{HIGH}$ \\
\hline $\mathrm{TlNO}_{4}$ & $\mathrm{CdI} 2$ & $\mathrm{HIGH}$ \\
\hline $\mathrm{TlNS}_{4}$ & $\mathrm{CdI} 2$ & LOW \\
\hline $\mathrm{TlNSe}_{4}$ & $\mathrm{CdI} 2$ & LOW \\
\hline $\mathrm{TlNTe}_{4}$ & CdI2 & LOW \\
\hline $\mathrm{TlPO}_{4}$ & CdI2 & HIGH \\
\hline $\mathrm{TlPS}_{4}$ & $\mathrm{CdI} 2$ & HIGH \\
\hline
\end{tabular}




\begin{tabular}{|c|c|c|}
\hline $\mathrm{TlPSe}_{4}$ & CdI2 & HIGH \\
\hline $\mathrm{TlPTe}_{4}$ & CdI2 & HIGH \\
\hline $\mathrm{TlAsO}_{4}$ & $\mathrm{CdI} 2$ & HIGH \\
\hline $\mathrm{TlAsS}_{4}$ & $\mathrm{CdI} 2$ & HIGH \\
\hline $\mathrm{TlAsSe}_{4}$ & $\mathrm{CdI} 2$ & HIGH \\
\hline $\mathrm{TlAsTe}_{4}$ & $\mathrm{CdI} 2$ & HIGH \\
\hline $\mathrm{TlSbO}_{4}$ & $\mathrm{CdI} 2$ & HIGH \\
\hline $\mathrm{TlSbS}_{4}$ & CdI2 & HIGH \\
\hline $\mathrm{TlSbSe}_{4}$ & CdI2 & HIGH \\
\hline $\mathrm{TlSbTe}_{4}$ & $\mathrm{CdI} 2$ & HIGH \\
\hline $\mathrm{TlBiO}_{4}$ & $\mathrm{CdI} 2$ & HIGH \\
\hline $\mathrm{TlBiS}_{4}$ & $\mathrm{CdI} 2$ & HIGH \\
\hline $\mathrm{TlBiSe}_{4}$ & CdI2 & HIGH \\
\hline $\mathrm{TlBiTe}_{4}$ & CdI2 & HIGH \\
\hline $\mathrm{BNO}_{4}$ & $\mathrm{PdS} 2$ & MEDIUM \\
\hline $\mathrm{BNS}_{4}$ & $\mathrm{PdS} 2$ & LOW \\
\hline $\mathrm{BNSe}_{4}$ & $\mathrm{PdS} 2$ & LOW \\
\hline $\mathrm{BNTe}_{4}$ & $\mathrm{PdS} 2$ & MEDIUM \\
\hline $\mathrm{BPO}_{4}$ & $\mathrm{PdS} 2$ & MEDIUM \\
\hline $\mathrm{BPS}_{4}$ & $\mathrm{PdS} 2$ & LOW \\
\hline $\mathrm{BPSe}_{4}$ & $\mathrm{PdS} 2$ & LOW \\
\hline $\mathrm{BPTe}_{4}$ & $\mathrm{PdS} 2$ & LOW \\
\hline $\mathrm{BAsO}_{4}$ & PdS2 & MEDIUM \\
\hline $\mathrm{BAsS}_{4}$ & $\mathrm{PdS} 2$ & LOW \\
\hline $\mathrm{BAsSe}_{4}$ & $\mathrm{PdS} 2$ & LOW \\
\hline $\mathrm{BAsTe}_{4}$ & $\mathrm{PdS} 2$ & LOW \\
\hline $\mathrm{BSbO}_{4}$ & $\mathrm{PdS} 2$ & MEDIUM \\
\hline
\end{tabular}




\begin{tabular}{|c|c|c|}
\hline $\mathrm{BSbS}_{4}$ & PdS2 & HIGH \\
\hline $\mathrm{BSbSe}_{4}$ & PdS2 & LOW \\
\hline $\mathrm{BSbTe}_{4}$ & PdS2 & LOW \\
\hline $\mathrm{BBiO}_{4}$ & $\mathrm{PdS} 2$ & MEDIUM \\
\hline $\mathrm{BBiS}_{4}$ & PdS2 & HIGH \\
\hline $\mathrm{BBiSe}_{4}$ & $\mathrm{PdS} 2$ & LOW \\
\hline $\mathrm{BBiTe}_{4}$ & $\mathrm{PdS} 2$ & LOW \\
\hline $\mathrm{AlNO}_{4}$ & PdS2 & MEDIUM \\
\hline $\mathrm{AlNS}_{4}$ & $\mathrm{PdS} 2$ & MEDIUM \\
\hline $\mathrm{AlNSe}_{4}$ & PdS2 & MEDIUM \\
\hline $\mathrm{AlNTe}_{4}$ & PdS2 & LOW \\
\hline $\mathrm{AlPO}_{4}$ & $\mathrm{PdS} 2$ & HIGH \\
\hline $\mathrm{AlPS}_{4}$ & PdS2 & HIGH \\
\hline $\mathrm{AlPSe}_{4}$ & PdS2 & HIGH \\
\hline $\mathrm{AlPTe}_{4}$ & PdS2 & HIGH \\
\hline $\mathrm{AlAsO}_{4}$ & PdS2 & HIGH \\
\hline $\mathrm{AlAsS}_{4}$ & PdS2 & HIGH \\
\hline $\mathrm{AlAsSe}_{4}$ & PdS2 & HIGH \\
\hline $\mathrm{AlAsTe}_{4}$ & $\mathrm{PdS} 2$ & HIGH \\
\hline $\mathrm{AlSbO}_{4}$ & PdS2 & HIGH \\
\hline $\mathrm{AlSbS}_{4}$ & $\mathrm{PdS} 2$ & HIGH \\
\hline $\mathrm{AlSbSe}_{4}$ & PdS2 & HIGH \\
\hline $\mathrm{AlSbTe}_{4}$ & PdS2 & HIGH \\
\hline $\mathrm{AlBiO}_{4}$ & $\mathrm{PdS} 2$ & HIGH \\
\hline $\mathrm{AlBiS}_{4}$ & PdS2 & HIGH \\
\hline $\mathrm{AlBiSe}_{4}$ & $\mathrm{PdS} 2$ & HIGH \\
\hline $\mathrm{AlBiTe}_{4}$ & PdS2 & HIGH \\
\hline
\end{tabular}




\begin{tabular}{|c|c|c|}
\hline $\mathrm{GaNO}_{4}$ & $\mathrm{PdS} 2$ & HIGH \\
\hline $\mathrm{GaNS}_{4}$ & $\mathrm{PdS} 2$ & MEDIUM \\
\hline $\mathrm{GaNSe}_{4}$ & $\mathrm{PdS} 2$ & LOW \\
\hline $\mathrm{GaNTe}_{4}$ & $\mathrm{PdS} 2$ & LOW \\
\hline $\mathrm{GaPO}_{4}$ & $\mathrm{PdS} 2$ & HIGH \\
\hline $\mathrm{GaPS}_{4}$ & $\mathrm{PdS} 2$ & HIGH \\
\hline $\mathrm{GaPSe}_{4}$ & $\mathrm{PdS} 2$ & HIGH \\
\hline $\mathrm{GaPTe}_{4}$ & $\mathrm{PdS} 2$ & HIGH \\
\hline $\mathrm{GaAsO}_{4}$ & $\mathrm{PdS} 2$ & HIGH \\
\hline $\mathrm{GaAsS}_{4}$ & $\mathrm{PdS} 2$ & HIGH \\
\hline $\mathrm{GaAsSe}_{4}$ & $\mathrm{PdS} 2$ & HIGH \\
\hline $\mathrm{GaAsTe}_{4}$ & $\mathrm{PdS} 2$ & LOW \\
\hline $\mathrm{GaSbO}_{4}$ & $\mathrm{PdS} 2$ & MEDIUM \\
\hline $\mathrm{GaSbS}_{4}$ & $\mathrm{PdS} 2$ & HIGH \\
\hline $\mathrm{GaSbSe}_{4}$ & $\mathrm{PdS} 2$ & HIGH \\
\hline $\mathrm{GaSbTe}_{4}$ & $\mathrm{PdS} 2$ & LOW \\
\hline $\mathrm{GaBiO}_{4}$ & $\mathrm{PdS} 2$ & HIGH \\
\hline $\mathrm{GaBiS}_{4}$ & $\mathrm{PdS} 2$ & HIGH \\
\hline $\mathrm{GaBiSe}_{4}$ & $\mathrm{PdS} 2$ & HIGH \\
\hline $\mathrm{GaBiTe}_{4}$ & $\mathrm{PdS} 2$ & HIGH \\
\hline $\mathrm{InNO}_{4}$ & $\mathrm{PdS} 2$ & MEDIUM \\
\hline $\mathrm{InNS}_{4}$ & $\mathrm{PdS} 2$ & LOW \\
\hline $\mathrm{InNSe}_{4}$ & $\mathrm{PdS} 2$ & LOW \\
\hline $\mathrm{InNTe}_{4}$ & $\mathrm{PdS} 2$ & LOW \\
\hline $\mathrm{InPO}_{4}$ & $\mathrm{PdS} 2$ & HIGH \\
\hline $\mathrm{InPS}_{4}$ & PdS2 & $\mathrm{HIGH}$ \\
\hline $\mathrm{InPSe}_{4}$ & $\mathrm{PdS} 2$ & HIGH \\
\hline
\end{tabular}




\begin{tabular}{|c|c|c|}
\hline $\mathrm{InPTe}_{4}$ & $\mathrm{PdS} 2$ & $\mathrm{HIGH}$ \\
\hline $\mathrm{InAsO}_{4}$ & $\mathrm{PdS} 2$ & $\mathrm{HIGH}$ \\
\hline $\operatorname{InAsS}_{4}$ & $\mathrm{PdS} 2$ & $\mathrm{HIGH}$ \\
\hline $\mathrm{InAsSe}_{4}$ & $\mathrm{PdS} 2$ & $\mathrm{HIGH}$ \\
\hline $\mathrm{InAsTe}_{4}$ & $\mathrm{PdS} 2$ & $\mathrm{HIGH}$ \\
\hline $\mathrm{InSbO}_{4}$ & $\mathrm{PdS} 2$ & $\mathrm{HIGH}$ \\
\hline $\mathrm{InSbS}_{4}$ & PdS2 & $\mathrm{HIGH}$ \\
\hline $\mathrm{InSbSe}_{4}$ & $\mathrm{PdS} 2$ & $\mathrm{HIGH}$ \\
\hline $\mathrm{InSbTe}_{4}$ & $\mathrm{PdS} 2$ & $\mathrm{HIGH}$ \\
\hline $\mathrm{InBiO}_{4}$ & $\mathrm{PdS} 2$ & $\mathrm{HIGH}$ \\
\hline $\mathrm{InBiS}_{4}$ & $\mathrm{PdS} 2$ & $\mathrm{HIGH}$ \\
\hline $\mathrm{InBiSe}_{4}$ & $\mathrm{PdS} 2$ & $\mathrm{HIGH}$ \\
\hline $\mathrm{InBiTe}_{4}$ & $\mathrm{PdS} 2$ & $\mathrm{HIGH}$ \\
\hline $\mathrm{TlNO}_{4}$ & $\mathrm{PdS} 2$ & $\mathrm{HIGH}$ \\
\hline $\mathrm{TlNS}_{4}$ & $\mathrm{PdS} 2$ & LOW \\
\hline $\mathrm{TlNSe}_{4}$ & PdS2 & LOW \\
\hline $\mathrm{TlNTe}_{4}$ & PdS2 & LOW \\
\hline $\mathrm{TlPO}_{4}$ & $\mathrm{PdS} 2$ & $\mathrm{HIGH}$ \\
\hline $\mathrm{TlPS}_{4}$ & $\mathrm{PdS} 2$ & $\mathrm{HIGH}$ \\
\hline $\mathrm{TlPSe}_{4}$ & PdS2 & $\mathrm{HIGH}$ \\
\hline $\mathrm{TlPTe}_{4}$ & $\mathrm{PdS} 2$ & HIGH \\
\hline $\mathrm{TlAsO}_{4}$ & $\mathrm{PdS} 2$ & $\mathrm{HIGH}$ \\
\hline $\mathrm{TlAsS}_{4}$ & PdS2 & $\mathrm{HIGH}$ \\
\hline $\mathrm{TlAsSe}_{4}$ & $\mathrm{PdS} 2$ & $\mathrm{HIGH}$ \\
\hline $\mathrm{TlAsTe}_{4}$ & $\mathrm{PdS} 2$ & $\mathrm{HIGH}$ \\
\hline $\mathrm{TlSbO}_{4}$ & $\mathrm{PdS} 2$ & $\mathrm{HIGH}$ \\
\hline $\mathrm{TlSbS}_{4}$ & $\mathrm{PdS} 2$ & HIGH \\
\hline
\end{tabular}




\begin{tabular}{|c|c|c|}
\hline $\mathrm{TlSbSe}_{4}$ & PdS2 & HIGH \\
\hline $\mathrm{TlSbTe}_{4}$ & PdS2 & HIGH \\
\hline $\mathrm{TlBiO}_{4}$ & PdS2 & HIGH \\
\hline $\mathrm{TlBiS}_{4}$ & PdS2 & HIGH \\
\hline $\mathrm{TlBiSe}_{4}$ & PdS2 & HIGH \\
\hline $\mathrm{TlBiTe}_{4}$ & $\mathrm{PdS} 2$ & HIGH \\
\hline $\mathrm{BNO}_{4}$ & ReS2 & MEDIUM \\
\hline $\mathrm{BNS}_{4}$ & ReS2 & LOW \\
\hline $\mathrm{BNSe}_{4}$ & ReS2 & LOW \\
\hline $\mathrm{BNTe}_{4}$ & $\operatorname{ReS} 2$ & MEDIUM \\
\hline $\mathrm{BPO}_{4}$ & $\operatorname{ReS} 2$ & MEDIUM \\
\hline $\mathrm{BPS}_{4}$ & ReS2 & LOW \\
\hline $\mathrm{BPSe}_{4}$ & ReS2 & LOW \\
\hline $\mathrm{BPTe}_{4}$ & ReS2 & LOW \\
\hline $\mathrm{BAsO}_{4}$ & $\operatorname{ReS} 2$ & MEDIUM \\
\hline $\mathrm{BAsS}_{4}$ & ReS2 & LOW \\
\hline $\mathrm{BAsSe}_{4}$ & ReS2 & LOW \\
\hline $\mathrm{BAsTe}_{4}$ & ReS2 & LOW \\
\hline $\mathrm{BSbO}_{4}$ & $\operatorname{ReS} 2$ & MEDIUM \\
\hline $\mathrm{BSbS}_{4}$ & $\operatorname{ReS} 2$ & HIGH \\
\hline $\mathrm{BSbSe}_{4}$ & ReS2 & LOW \\
\hline $\mathrm{BSbTe}_{4}$ & ReS2 & LOW \\
\hline $\mathrm{BBiO}_{4}$ & ReS2 & MEDIUM \\
\hline $\mathrm{BBiS}_{4}$ & ReS2 & HIGH \\
\hline $\mathrm{BBiSe}_{4}$ & $\operatorname{ReS} 2$ & LOW \\
\hline $\mathrm{BBiTe}_{4}$ & $\operatorname{ReS} 2$ & LOW \\
\hline $\mathrm{AlNO}_{4}$ & ReS2 & MEDIUM \\
\hline
\end{tabular}




\begin{tabular}{|c|c|c|}
\hline $\mathrm{AlNS}_{4}$ & ReS2 & MEDIUM \\
\hline $\mathrm{AlNSe}_{4}$ & ReS2 & MEDIUM \\
\hline $\mathrm{AlNTe}_{4}$ & ReS2 & LOW \\
\hline $\mathrm{AlPO}_{4}$ & ReS2 & $\mathrm{HIGH}$ \\
\hline $\mathrm{AlPS}_{4}$ & ReS2 & HIGH \\
\hline $\mathrm{AlPSe}_{4}$ & ReS2 & HIGH \\
\hline $\mathrm{AlPTe}_{4}$ & ReS2 & HIGH \\
\hline $\mathrm{AlAsO}_{4}$ & ReS2 & HIGH \\
\hline $\mathrm{AlAsS}_{4}$ & ReS2 & HIGH \\
\hline $\mathrm{AlAsSe}_{4}$ & ReS2 & HIGH \\
\hline $\mathrm{AlAsTe}_{4}$ & ReS2 & HIGH \\
\hline $\mathrm{AlSbO}_{4}$ & ReS2 & HIGH \\
\hline $\mathrm{AlSbS}_{4}$ & ReS2 & HIGH \\
\hline $\mathrm{AlSbSe}_{4}$ & ReS2 & HIGH \\
\hline $\mathrm{AlSbTe}_{4}$ & ReS2 & HIGH \\
\hline $\mathrm{AlBiO}_{4}$ & ReS2 & HIGH \\
\hline $\mathrm{AlBiS}_{4}$ & ReS2 & HIGH \\
\hline $\mathrm{AlBiSe}_{4}$ & ReS2 & HIGH \\
\hline $\mathrm{AlBiTe}_{4}$ & ReS2 & HIGH \\
\hline $\mathrm{GaNO}_{4}$ & ReS2 & HIGH \\
\hline $\mathrm{GaNS}_{4}$ & ReS2 & MEDIUM \\
\hline $\mathrm{GaNSe}_{4}$ & ReS2 & LOW \\
\hline $\mathrm{GaNTe}_{4}$ & ReS2 & LOW \\
\hline $\mathrm{GaPO}_{4}$ & ReS2 & HIGH \\
\hline $\mathrm{GaPS}_{4}$ & ReS2 & HIGH \\
\hline $\mathrm{GaPSe}_{4}$ & ReS2 & HIGH \\
\hline $\mathrm{GaPTe}_{4}$ & ReS2 & HIGH \\
\hline
\end{tabular}




\begin{tabular}{|c|c|c|}
\hline $\mathrm{GaAsO}_{4}$ & ReS2 & $\mathrm{HIGH}$ \\
\hline $\mathrm{GaAsS}_{4}$ & ReS2 & $\mathrm{HIGH}$ \\
\hline $\mathrm{GaAsSe}_{4}$ & ReS2 & $\mathrm{HIGH}$ \\
\hline $\mathrm{GaAsTe}_{4}$ & ReS2 & $\mathrm{HIGH}$ \\
\hline $\mathrm{GaSbO}_{4}$ & ReS2 & $\mathrm{HIGH}$ \\
\hline $\mathrm{GaSbS}_{4}$ & ReS2 & $\mathrm{HIGH}$ \\
\hline $\mathrm{GaSbSe}_{4}$ & ReS2 & $\mathrm{HIGH}$ \\
\hline $\mathrm{GaSbTe}_{4}$ & ReS2 & $\mathrm{HIGH}$ \\
\hline $\mathrm{GaBiO}_{4}$ & ReS2 & $\mathrm{HIGH}$ \\
\hline $\mathrm{GaBiS}_{4}$ & ReS2 & $\mathrm{HIGH}$ \\
\hline $\mathrm{GaBiSe}_{4}$ & ReS2 & HIGH \\
\hline $\mathrm{GaBiTe}_{4}$ & ReS2 & HIGH \\
\hline $\mathrm{InNO}_{4}$ & ReS2 & $\mathrm{HIGH}$ \\
\hline $\mathrm{InNS}_{4}$ & ReS2 & LOW \\
\hline $\mathrm{InNSe}_{4}$ & ReS2 & LOW \\
\hline $\mathrm{InNTe}_{4}$ & ReS2 & LOW \\
\hline $\mathrm{InPO}_{4}$ & ReS2 & $\mathrm{HIGH}$ \\
\hline $\mathrm{InPS}_{4}$ & $\mathrm{ReS} 2$ & $\mathrm{HIGH}$ \\
\hline $\mathrm{InPSe}_{4}$ & ReS2 & $\mathrm{HIGH}$ \\
\hline $\mathrm{InPTe}_{4}$ & ReS2 & $\mathrm{HIGH}$ \\
\hline $\mathrm{InAsO}_{4}$ & ReS2 & $\mathrm{HIGH}$ \\
\hline $\mathrm{InAsS}_{4}$ & ReS2 & $\mathrm{HIGH}$ \\
\hline $\mathrm{InAsSe}_{4}$ & ReS2 & $\mathrm{HIGH}$ \\
\hline $\mathrm{InAsTe}_{4}$ & ReS2 & HIGH \\
\hline $\mathrm{InSbO}_{4}$ & ReS2 & HIGH \\
\hline $\mathrm{InSbS}_{4}$ & ReS2 & $\mathrm{HIG}$ \\
\hline $\mathrm{InSbSe}_{4}$ & ReS2 & $\mathrm{HIG}$ \\
\hline
\end{tabular}




\begin{tabular}{|c|c|c|}
\hline $\mathrm{InSbTe}_{4}$ & ReS2 & $\mathrm{HIGH}$ \\
\hline $\mathrm{InBiO}_{4}$ & ReS2 & $\mathrm{HIGH}$ \\
\hline $\mathrm{InBiS}_{4}$ & ReS2 & $\mathrm{HIGH}$ \\
\hline $\mathrm{InBiSe}_{4}$ & ReS2 & $\mathrm{HIGH}$ \\
\hline $\mathrm{InBiTe}_{4}$ & ReS2 & $\mathrm{HIGH}$ \\
\hline $\mathrm{TlNO}_{4}$ & ReS2 & $\mathrm{HIGH}$ \\
\hline $\mathrm{TlNS}_{4}$ & ReS2 & LOW \\
\hline $\mathrm{TlNSe}_{4}$ & ReS2 & LOW \\
\hline $\mathrm{TlNTe}_{4}$ & ReS2 & LOW \\
\hline $\mathrm{TlPO}_{4}$ & ReS2 & $\mathrm{HIGH}$ \\
\hline $\mathrm{TlPS}_{4}$ & ReS2 & $\mathrm{HIGH}$ \\
\hline $\mathrm{TlPSe}_{4}$ & ReS2 & $\mathrm{HIGH}$ \\
\hline $\mathrm{TlPTe}_{4}$ & ReS2 & $\mathrm{HIGH}$ \\
\hline $\mathrm{TlAsO}_{4}$ & ReS2 & $\mathrm{HIGH}$ \\
\hline $\mathrm{TlAsS}_{4}$ & ReS2 & $\mathrm{HIGH}$ \\
\hline $\mathrm{TlAsSe}_{4}$ & ReS2 & $\mathrm{HIGH}$ \\
\hline $\mathrm{TlAsTe}_{4}$ & ReS2 & $\mathrm{HIGH}$ \\
\hline $\mathrm{TlSbO}_{4}$ & ReS2 & $\mathrm{HIGH}$ \\
\hline $\mathrm{TlSbS}_{4}$ & ReS2 & $\mathrm{HIGH}$ \\
\hline $\mathrm{TlSbSe}_{4}$ & ReS2 & $\mathrm{HIGH}$ \\
\hline $\mathrm{TlSbTe}_{4}$ & ReS2 & $\mathrm{HIGH}$ \\
\hline $\mathrm{TlBiO}_{4}$ & ReS2 & $\mathrm{HIGH}$ \\
\hline $\mathrm{TlBiS}_{4}$ & ReS2 & $\mathrm{HIGH}$ \\
\hline $\mathrm{TlBiSe}_{4}$ & ReS2 & $\mathrm{HIGH}$ \\
\hline $\mathrm{TlBiTe}_{4}$ & ReS2 & $\mathrm{HIGH}$ \\
\hline $\mathrm{BNO}_{2}$ & $\mathrm{RhO}$ & LOW \\
\hline $\mathrm{BNS}_{2}$ & $\mathrm{RhO}$ & LOW \\
\hline
\end{tabular}




\begin{tabular}{|c|c|c|}
\hline $\mathrm{BNSe}_{2}$ & $\mathrm{RhO}$ & LOW \\
\hline $\mathrm{BNTe}_{2}$ & $\mathrm{RhO}$ & MEDIUM \\
\hline $\mathrm{BPO}_{2}$ & $\mathrm{RhO}$ & MEDIUM \\
\hline $\mathrm{BPS}_{2}$ & $\mathrm{RhO}$ & LOW \\
\hline $\mathrm{BPSe}_{2}$ & $\mathrm{RhO}$ & LOW \\
\hline $\mathrm{BPTe}_{2}$ & $\mathrm{RhO}$ & LOW \\
\hline $\mathrm{BAsO}_{2}$ & $\mathrm{RhO}$ & MEDIUM \\
\hline $\mathrm{BAsS}_{2}$ & $\mathrm{RhO}$ & LOW \\
\hline $\mathrm{BAsSe}_{2}$ & $\mathrm{RhO}$ & LOW \\
\hline $\mathrm{BAsTe}_{2}$ & $\mathrm{RhO}$ & LOW \\
\hline $\mathrm{BSbO}_{2}$ & $\mathrm{RhO}$ & MEDIUM \\
\hline $\mathrm{BSbS}_{2}$ & $\mathrm{RhO}$ & LOW \\
\hline $\mathrm{BSbSe}_{2}$ & $\mathrm{RhO}$ & LOW \\
\hline $\mathrm{BSbTe}_{2}$ & $\mathrm{RhO}$ & LOW \\
\hline $\mathrm{BBiO}_{2}$ & $\mathrm{RhO}$ & MEDIUM \\
\hline $\mathrm{BBiS}_{2}$ & $\mathrm{RhO}$ & LOW \\
\hline $\mathrm{BBiSe}_{2}$ & $\mathrm{RhO}$ & LOW \\
\hline $\mathrm{BBiTe}_{2}$ & $\mathrm{RhO}$ & LOW \\
\hline $\mathrm{AlNO}_{2}$ & $\mathrm{RhO}$ & MEDIUM \\
\hline $\mathrm{AlNS}_{2}$ & $\mathrm{RhO}$ & MEDIUM \\
\hline $\mathrm{AlNSe}_{2}$ & $\mathrm{RhO}$ & MEDIUM \\
\hline $\mathrm{AlNTe}_{2}$ & $\mathrm{RhO}$ & LOW \\
\hline $\mathrm{AlPO}_{2}$ & $\mathrm{RhO}$ & MEDIUM \\
\hline $\mathrm{AlPS}_{2}$ & $\mathrm{RhO}$ & HIGH \\
\hline $\mathrm{AlPSe}_{2}$ & $\mathrm{RhO}$ & HIGH \\
\hline $\mathrm{AlPTe}_{2}$ & $\mathrm{RhO}$ & HIGH \\
\hline $\mathrm{AlAsO}_{2}$ & $\mathrm{RhO}$ & MEDIUM \\
\hline
\end{tabular}




\begin{tabular}{|c|c|c|}
\hline $\mathrm{AlAsS}_{2}$ & $\mathrm{RhO}$ & HIGH \\
\hline $\mathrm{AlAsSe}_{2}$ & $\mathrm{RhO}$ & HIGH \\
\hline $\mathrm{AlAsTe}_{2}$ & $\mathrm{RhO}$ & LOW \\
\hline $\mathrm{AlSbO}_{2}$ & $\mathrm{RhO}$ & MEDIUM \\
\hline $\mathrm{AlSbS}_{2}$ & $\mathrm{RhO}$ & HIGH \\
\hline $\mathrm{AlSbSe}_{2}$ & $\mathrm{RhO}$ & HIGH \\
\hline $\mathrm{AlSbTe}_{2}$ & $\mathrm{RhO}$ & LOW \\
\hline $\mathrm{AlBiO}_{2}$ & $\mathrm{RhO}$ & MEDIUM \\
\hline $\mathrm{AlBiS}_{2}$ & $\mathrm{RhO}$ & HIGH \\
\hline $\mathrm{AlBiSe}_{2}$ & $\mathrm{RhO}$ & HIGH \\
\hline $\mathrm{AlBiTe}_{2}$ & $\mathrm{RhO}$ & HIGH \\
\hline $\mathrm{GaNO}_{2}$ & $\mathrm{RhO}$ & MEDIUM \\
\hline $\mathrm{GaNS}_{2}$ & $\mathrm{RhO}$ & MEDIUM \\
\hline $\mathrm{GaNSe}_{2}$ & $\mathrm{RhO}$ & LOW \\
\hline $\mathrm{GaNTe}_{2}$ & $\mathrm{RhO}$ & LOW \\
\hline $\mathrm{GaPO}_{2}$ & $\mathrm{RhO}$ & MEDIUM \\
\hline $\mathrm{GaPS}_{2}$ & $\mathrm{RhO}$ & HIGH \\
\hline $\mathrm{GaPSe}_{2}$ & $\mathrm{RhO}$ & HIGH \\
\hline $\mathrm{GaPTe}_{2}$ & $\mathrm{RhO}$ & LOW \\
\hline $\mathrm{GaAsO}_{2}$ & $\mathrm{RhO}$ & MEDIUM \\
\hline $\mathrm{GaAsS}_{2}$ & $\mathrm{RhO}$ & MEDIUM \\
\hline $\mathrm{GaAsSe}_{2}$ & $\mathrm{RhO}$ & LOW \\
\hline $\mathrm{GaAsTe}_{2}$ & $\mathrm{RhO}$ & LOW \\
\hline $\mathrm{GaSbO}_{2}$ & $\mathrm{RhO}$ & MEDIUM \\
\hline $\mathrm{GaSbS}_{2}$ & $\mathrm{RhO}$ & MEDIUM \\
\hline $\mathrm{GaSbSe}_{2}$ & $\mathrm{RhO}$ & LOW \\
\hline $\mathrm{GaSbTe}_{2}$ & $\mathrm{RhO}$ & LOW \\
\hline
\end{tabular}




\begin{tabular}{|c|c|c|}
\hline $\mathrm{GaBiO}_{2}$ & $\mathrm{RhO}$ & MEDIUM \\
\hline $\mathrm{GaBiS}_{2}$ & $\mathrm{RhO}$ & MEDIUM \\
\hline $\mathrm{GaBiSe}_{2}$ & $\mathrm{RhO}$ & MEDIUM \\
\hline $\mathrm{GaBiTe}_{2}$ & $\mathrm{RhO}$ & LOW \\
\hline $\mathrm{InNO}_{2}$ & $\mathrm{RhO}$ & MEDIUM \\
\hline $\operatorname{InNS}_{2}$ & $\mathrm{RhO}$ & LOW \\
\hline $\operatorname{InNSe}_{2}$ & $\mathrm{RhO}$ & LOW \\
\hline $\mathrm{InNTe}_{2}$ & $\mathrm{RhO}$ & LOW \\
\hline $\mathrm{InPO}_{2}$ & $\mathrm{RhO}$ & MEDIUM \\
\hline $\mathrm{InPS}_{2}$ & $\mathrm{RhO}$ & MEDIUM \\
\hline $\mathrm{InPSe}_{2}$ & $\mathrm{RhO}$ & HIGH \\
\hline $\mathrm{InPTe}_{2}$ & $\mathrm{RhO}$ & LOW \\
\hline $\mathrm{InAsO}_{2}$ & $\mathrm{RhO}$ & MEDIUM \\
\hline $\mathrm{InAsS}_{2}$ & $\mathrm{RhO}$ & MEDIUM \\
\hline $\mathrm{InAsSe}_{2}$ & $\mathrm{RhO}$ & MEDIUM \\
\hline $\operatorname{InAsTe}_{2}$ & $\mathrm{RhO}$ & LOW \\
\hline $\mathrm{InSbO}_{2}$ & $\mathrm{RhO}$ & MEDIUM \\
\hline $\mathrm{InSbS}_{2}$ & $\mathrm{RhO}$ & MEDIUM \\
\hline $\mathrm{InSbSe}_{2}$ & $\mathrm{RhO}$ & MEDIUM \\
\hline $\mathrm{InSbTe}_{2}$ & $\mathrm{RhO}$ & LOW \\
\hline $\mathrm{InBiO}_{2}$ & $\mathrm{RhO}$ & MEDIUM \\
\hline $\mathrm{InBiS}_{2}$ & $\mathrm{RhO}$ & MEDIUM \\
\hline $\mathrm{InBiSe}_{2}$ & $\mathrm{RhO}$ & HIGH \\
\hline $\mathrm{InBiTe}_{2}$ & $\mathrm{RhO}$ & LOW \\
\hline $\mathrm{TlNO}_{2}$ & $\mathrm{RhO}$ & MEDIUM \\
\hline $\mathrm{TlNS}_{2}$ & $\mathrm{RhO}$ & LOW \\
\hline $\mathrm{TlNSe}_{2}$ & $\mathrm{RhO}$ & LOW \\
\hline
\end{tabular}




\begin{tabular}{|c|c|c|}
\hline $\mathrm{TlNTe}_{2}$ & $\mathrm{RhO}$ & LOW \\
\hline $\mathrm{TlPO}_{2}$ & $\mathrm{RhO}$ & MEDIUM \\
\hline $\mathrm{TlPS}_{2}$ & $\mathrm{RhO}$ & HIGH \\
\hline $\mathrm{TlPSe}_{2}$ & $\mathrm{RhO}$ & HIGH \\
\hline $\mathrm{TlPTe}_{2}$ & $\mathrm{RhO}$ & LOW \\
\hline $\mathrm{TlAsO}_{2}$ & $\mathrm{RhO}$ & MEDIUM \\
\hline $\mathrm{TlAsS}_{2}$ & $\mathrm{RhO}$ & $\mathrm{HIGH}$ \\
\hline $\mathrm{TlAsSe}_{2}$ & $\mathrm{RhO}$ & HIGH \\
\hline $\mathrm{TlAsTe}_{2}$ & $\mathrm{RhO}$ & LOW \\
\hline $\mathrm{TlSbO}_{2}$ & $\mathrm{RhO}$ & MEDIUM \\
\hline $\mathrm{TlSbS}_{2}$ & $\mathrm{RhO}$ & HIGH \\
\hline $\mathrm{TlSbSe}_{2}$ & $\mathrm{RhO}$ & HIGH \\
\hline $\mathrm{TlSbTe}_{2}$ & $\mathrm{RhO}$ & LOW \\
\hline $\mathrm{TlBiO}_{2}$ & $\mathrm{RhO}$ & MEDIUM \\
\hline $\mathrm{TlBiS}_{2}$ & $\mathrm{RhO}$ & HIGH \\
\hline $\mathrm{TlBiSe}_{2}$ & $\mathrm{RhO}$ & HIGH \\
\hline $\mathrm{TlBiTe}_{2}$ & $\mathrm{RhO}$ & HIGH \\
\hline $\mathrm{BNO}_{2}$ & $\mathrm{PbSe}$ & MEDIUM \\
\hline $\mathrm{BNS}_{2}$ & $\mathrm{PbSe}$ & LOW \\
\hline $\mathrm{BNSe}_{2}$ & $\mathrm{PbSe}$ & LOW \\
\hline $\mathrm{BNTe}_{2}$ & $\mathrm{PbSe}$ & MEDIUM \\
\hline $\mathrm{BPO}_{2}$ & $\mathrm{PbSe}$ & MEDIUM \\
\hline $\mathrm{BPS}_{2}$ & PbSe & LOW \\
\hline $\mathrm{BPSe}_{2}$ & $\mathrm{PbSe}$ & LOW \\
\hline $\mathrm{BPTe}_{2}$ & $\mathrm{PbSe}$ & LOW \\
\hline $\mathrm{BAsO}_{2}$ & $\mathrm{PbSe}$ & MEDIUM \\
\hline $\mathrm{BAsS}_{2}$ & $\mathrm{PbSe}$ & LOW \\
\hline
\end{tabular}




\begin{tabular}{|c|c|c|}
\hline $\mathrm{BAsSe}_{2}$ & $\mathrm{PbSe}$ & LOW \\
\hline $\mathrm{BAsTe}_{2}$ & $\mathrm{PbSe}$ & LOW \\
\hline $\mathrm{BSbO}_{2}$ & $\mathrm{PbSe}$ & MEDIUM \\
\hline $\mathrm{BSbS}_{2}$ & $\mathrm{PbSe}$ & LOW \\
\hline $\mathrm{BSbSe}_{2}$ & $\mathrm{PbSe}$ & LOW \\
\hline $\mathrm{BSbTe}_{2}$ & $\mathrm{PbSe}$ & LOW \\
\hline $\mathrm{BBiO}_{2}$ & $\mathrm{PbSe}$ & MEDIUM \\
\hline $\mathrm{BBiS}_{2}$ & $\mathrm{PbSe}$ & LOW \\
\hline $\mathrm{BBiSe}_{2}$ & $\mathrm{PbSe}$ & LOW \\
\hline $\mathrm{BBiTe}_{2}$ & $\mathrm{PbSe}$ & LOW \\
\hline $\mathrm{AlNO}_{2}$ & $\mathrm{PbSe}$ & MEDIUM \\
\hline $\mathrm{AlNS}_{2}$ & $\mathrm{PbSe}$ & MEDIUM \\
\hline $\mathrm{AlNSe}_{2}$ & $\mathrm{PbSe}$ & MEDIUM \\
\hline $\mathrm{AlNTe}_{2}$ & $\mathrm{PbSe}$ & LOW \\
\hline $\mathrm{AlPO}_{2}$ & $\mathrm{PbSe}$ & MEDIUM \\
\hline $\mathrm{AlPS}_{2}$ & $\mathrm{PbSe}$ & HIGH \\
\hline $\mathrm{AlPSe}_{2}$ & $\mathrm{PbSe}$ & HIGH \\
\hline $\mathrm{AlPTe}_{2}$ & $\mathrm{PbSe}$ & HIGH \\
\hline $\mathrm{AlAsO}_{2}$ & $\mathrm{PbSe}$ & MEDIUM \\
\hline $\mathrm{AlAsS}_{2}$ & $\mathrm{PbSe}$ & HIGH \\
\hline $\mathrm{AlAsSe}_{2}$ & $\mathrm{PbSe}$ & HIGH \\
\hline $\mathrm{AlAsTe}_{2}$ & $\mathrm{PbSe}$ & LOW \\
\hline $\mathrm{AlSbO}_{2}$ & $\mathrm{PbSe}$ & MEDIUM \\
\hline $\mathrm{AlSbS}_{2}$ & $\mathrm{PbSe}$ & HIGH \\
\hline $\mathrm{AlSbSe}_{2}$ & $\mathrm{PbSe}$ & HIGH \\
\hline $\mathrm{AlSbTe}_{2}$ & $\mathrm{PbSe}$ & LOW \\
\hline $\mathrm{AlBiO}_{2}$ & $\mathrm{PbSe}$ & MEDIUM \\
\hline
\end{tabular}




\begin{tabular}{|c|c|c|}
\hline $\mathrm{AlBiS}_{2}$ & $\mathrm{PbSe}$ & HIGH \\
\hline $\mathrm{AlBiSe}_{2}$ & $\mathrm{PbSe}$ & HIGH \\
\hline $\mathrm{AlBiTe}_{2}$ & $\mathrm{PbSe}$ & LOW \\
\hline $\mathrm{GaNO}_{2}$ & $\mathrm{PbSe}$ & MEDIUM \\
\hline $\mathrm{GaNS}_{2}$ & $\mathrm{PbSe}$ & MEDIUM \\
\hline $\mathrm{GaNSe}_{2}$ & $\mathrm{PbSe}$ & LOW \\
\hline $\mathrm{GaNTe}_{2}$ & $\mathrm{PbSe}$ & LOW \\
\hline $\mathrm{GaPO}_{2}$ & $\mathrm{PbSe}$ & MEDIUM \\
\hline $\mathrm{GaPS}_{2}$ & $\mathrm{PbSe}$ & HIGH \\
\hline $\mathrm{GaPSe}_{2}$ & $\mathrm{PbSe}$ & HIGH \\
\hline $\mathrm{GaPTe}_{2}$ & $\mathrm{PbSe}$ & $\mathrm{HIGH}$ \\
\hline $\mathrm{GaAsO}_{2}$ & $\mathrm{PbSe}$ & MEDIUM \\
\hline $\mathrm{GaAsS}_{2}$ & $\mathrm{PbSe}$ & HIGH \\
\hline $\mathrm{GaAsSe}_{2}$ & $\mathrm{PbSe}$ & LOW \\
\hline $\mathrm{GaAsTe}_{2}$ & $\mathrm{PbSe}$ & LOW \\
\hline $\mathrm{GaSbO}_{2}$ & $\mathrm{PbSe}$ & MEDIUM \\
\hline $\mathrm{GaSbS}_{2}$ & $\mathrm{PbSe}$ & MEDIUM \\
\hline $\mathrm{GaSbSe}_{2}$ & $\mathrm{PbSe}$ & LOW \\
\hline $\mathrm{GaSbTe}_{2}$ & $\mathrm{PbSe}$ & LOW \\
\hline $\mathrm{GaBiO}_{2}$ & $\mathrm{PbSe}$ & MEDIUM \\
\hline $\mathrm{GaBiS}_{2}$ & $\mathrm{PbSe}$ & MEDIUM \\
\hline $\mathrm{GaBiSe}_{2}$ & $\mathrm{PbSe}$ & MEDIUM \\
\hline $\mathrm{GaBiTe}_{2}$ & $\mathrm{PbSe}$ & LOW \\
\hline $\mathrm{InNO}_{2}$ & $\mathrm{PbSe}$ & MEDIUM \\
\hline $\mathrm{InNS}_{2}$ & $\mathrm{PbSe}$ & LOW \\
\hline $\mathrm{InNSe}_{2}$ & $\mathrm{PbSe}$ & LOW \\
\hline $\mathrm{InNTe}_{2}$ & $\mathrm{PbSe}$ & LOW \\
\hline
\end{tabular}




\begin{tabular}{|c|c|c|}
\hline $\mathrm{InPO}_{2}$ & $\mathrm{PbSe}$ & MEDIUM \\
\hline $\mathrm{InPS}_{2}$ & $\mathrm{PbSe}$ & HIGH \\
\hline $\mathrm{InPSe}_{2}$ & $\mathrm{PbSe}$ & HIGH \\
\hline $\mathrm{InPTe}_{2}$ & $\mathrm{PbSe}$ & LOW \\
\hline $\mathrm{InAsO}_{2}$ & $\mathrm{PbSe}$ & MEDIUM \\
\hline $\operatorname{InAs} \mathrm{A}_{2}$ & $\mathrm{PbSe}$ & MEDIUM \\
\hline $\mathrm{InAsSe}_{2}$ & $\mathrm{PbSe}$ & MEDIUM \\
\hline $\mathrm{InAsTe}_{2}$ & $\mathrm{PbSe}$ & LOW \\
\hline $\mathrm{InSbO}_{2}$ & $\mathrm{PbSe}$ & MEDIUM \\
\hline $\mathrm{InSbS}_{2}$ & $\mathrm{PbSe}$ & MEDIUM \\
\hline $\mathrm{InSbSe}_{2}$ & $\mathrm{PbSe}$ & MEDIUM \\
\hline $\mathrm{InSbTe}_{2}$ & $\mathrm{PbSe}$ & LOW \\
\hline $\mathrm{InBiO}_{2}$ & $\mathrm{PbSe}$ & MEDIUM \\
\hline $\mathrm{InBiS}_{2}$ & $\mathrm{PbSe}$ & MEDIUM \\
\hline $\mathrm{InBiSe}_{2}$ & $\mathrm{PbSe}$ & MEDIUM \\
\hline $\mathrm{InBiTe}_{2}$ & $\mathrm{PbSe}$ & LOW \\
\hline $\mathrm{TlNO}_{2}$ & $\mathrm{PbSe}$ & MEDIUM \\
\hline $\mathrm{TlNS}_{2}$ & $\mathrm{PbSe}$ & LOW \\
\hline $\mathrm{TlNSe}_{2}$ & $\mathrm{PbSe}$ & LOW \\
\hline $\mathrm{TlNTe}_{2}$ & $\mathrm{PbSe}$ & LOW \\
\hline $\mathrm{TlPO}_{2}$ & $\mathrm{PbSe}$ & MEDIUM \\
\hline $\mathrm{TlPS}_{2}$ & $\mathrm{PbSe}$ & HIGH \\
\hline $\mathrm{TlPSe}_{2}$ & $\mathrm{PbSe}$ & HIGH \\
\hline $\mathrm{TlPTe}_{2}$ & $\mathrm{PbSe}$ & LOW \\
\hline $\mathrm{TlAsO}_{2}$ & $\mathrm{PbSe}$ & MEDIUM \\
\hline $\mathrm{TlAsS}_{2}$ & $\mathrm{PbSe}$ & HIGH \\
\hline $\mathrm{TlAsSe}_{2}$ & $\mathrm{PbSe}$ & HIGH \\
\hline
\end{tabular}




\begin{tabular}{|c|c|c|}
\hline $\mathrm{TlAsTe}_{2}$ & $\mathrm{PbSe}$ & LOW \\
\hline $\mathrm{TlSbO}_{2}$ & $\mathrm{PbSe}$ & MEDIUM \\
\hline $\mathrm{TlSbS}_{2}$ & $\mathrm{PbSe}$ & HIGH \\
\hline $\mathrm{TlSbSe}_{2}$ & $\mathrm{PbSe}$ & HIGH \\
\hline $\mathrm{TlSbTe}_{2}$ & $\mathrm{PbSe}$ & LOW \\
\hline $\mathrm{TlBiO}_{2}$ & $\mathrm{PbSe}$ & MEDIUM \\
\hline $\mathrm{TlBiS}_{2}$ & PbSe & HIGH \\
\hline $\mathrm{TlBiSe}_{2}$ & $\mathrm{PbSe}$ & HIGH \\
\hline $\mathrm{TlBiTe}_{2}$ & $\mathrm{PbSe}$ & LOW \\
\hline $\mathrm{BNO}_{2}$ & GaSe & MEDIUM \\
\hline $\mathrm{BNS}_{2}$ & GaSe & LOW \\
\hline $\mathrm{BNSe}_{2}$ & GaSe & LOW \\
\hline $\mathrm{BNTe}_{2}$ & GaSe & MEDIUM \\
\hline $\mathrm{BPO}_{2}$ & GaSe & MEDIUM \\
\hline $\mathrm{BPS}_{2}$ & GaSe & LOW \\
\hline $\mathrm{BPSe}_{2}$ & GaSe & LOW \\
\hline $\mathrm{BPTe}_{2}$ & GaSe & LOW \\
\hline $\mathrm{BAsO}_{2}$ & GaSe & MEDIUM \\
\hline $\mathrm{BAsS}_{2}$ & GaSe & LOW \\
\hline $\mathrm{BAsSe}_{2}$ & GaSe & LOW \\
\hline $\mathrm{BAsTe}_{2}$ & GaSe & LOW \\
\hline $\mathrm{BSbO}_{2}$ & GaSe & MEDIUM \\
\hline $\mathrm{BSbS}_{2}$ & GaSe & LOW \\
\hline $\mathrm{BSbSe}_{2}$ & GaSe & LOW \\
\hline $\mathrm{BSbTe}_{2}$ & GaSe & LOW \\
\hline $\mathrm{BBiO}_{2}$ & GaSe & MEDIUM \\
\hline $\mathrm{BBiS}_{2}$ & GaSe & LOW \\
\hline
\end{tabular}




$\begin{array}{lcc}\mathrm{BBiSe}_{2} & \text { GaSe } & \text { LOW } \\ \mathrm{BBiTe}_{2} & \text { GaSe } & \text { LOW } \\ \mathrm{AlNO}_{2} & \text { GaSe } & \text { MEDIUM } \\ \mathrm{AlNS}_{2} & \text { GaSe } & \text { MEDIUM } \\ \mathrm{AlNSe}_{2} & \text { GaSe } & \text { MEDIUM } \\ \mathrm{AlNTe}_{2} & \text { GaSe } & \text { LOW } \\ \mathrm{AlPO}_{2} & \text { GaSe } & \text { MEDIUM } \\ \mathrm{AlPS}_{2} & \text { GaSe } & \text { HIGH } \\ \mathrm{AlPSe}_{2} & \text { GaSe } & \text { HIGH } \\ \mathrm{AlPTe}_{2} & \text { GaSe } & \text { HIGH } \\ \mathrm{AlAsO}_{2} & \text { GaSe } & \text { MEDIUM } \\ \mathrm{AlAsS}_{2} & \text { GaSe } & \text { HIGH } \\ \mathrm{AlAsSe}_{2} & \text { GaSe } & \text { HIGH } \\ \mathrm{AlAsTe}_{2} & \text { GaSe } & \text { HIGH } \\ \mathrm{AlSbO}_{2} & \text { GaSe } & \text { MEDIUM } \\ \mathrm{AlSbS}_{2} & \text { GaSe } & \text { HIGH } \\ \mathrm{AlSbSe}_{2} & \text { GaSe } & \text { HIGH } \\ \mathrm{AlSbTe}_{2} & \text { GaSe } & \text { HIGH } \\ \mathrm{AlBiO}_{2} & \text { GaSe } & \text { MEDIUM } \\ \mathrm{AlBiS}_{2} & \text { GaSe } & \text { HIGH } \\ \mathrm{AlBiSe}_{2} & \text { GaSe } & \text { HIGH } \\ \mathrm{AlBiTe}_{2} & \text { GaSe } & \text { HIGH } \\ \mathrm{GaNO}_{2} & \text { GaSe } & \text { MEDIUM } \\ \mathrm{GaNS}_{2} & \text { GaSe } & \text { MEDIUM } \\ \mathrm{GaNSe}_{2} & \text { GaSe } & \text { LOW } \\ \mathrm{GaNTe}_{2} & \text { GaSe } & \text { LOW } \\ 2 & \text { GaSe } & \text { HIGH }\end{array}$




\begin{tabular}{|c|c|c|}
\hline $\mathrm{GaPS}_{2}$ & GaSe & HIGH \\
\hline $\mathrm{GaPSe}_{2}$ & GaSe & HIGH \\
\hline $\mathrm{GaPTe}_{2}$ & GaSe & HIGH \\
\hline $\mathrm{GaAsO}_{2}$ & GaSe & MEDIUM \\
\hline $\mathrm{GaAsS}_{2}$ & GaSe & HIGH \\
\hline $\mathrm{GaAsSe}_{2}$ & GaSe & HIGH \\
\hline $\mathrm{GaAsTe}_{2}$ & GaSe & HIGH \\
\hline $\mathrm{GaSbO}_{2}$ & GaSe & MEDIUM \\
\hline $\mathrm{GaSbS}_{2}$ & GaSe & HIGH \\
\hline $\mathrm{GaSbSe}_{2}$ & GaSe & HIGH \\
\hline $\mathrm{GaSbTe}_{2}$ & GaSe & HIGH \\
\hline $\mathrm{GaBiO}_{2}$ & GaSe & MEDIUM \\
\hline $\mathrm{GaBiS}_{2}$ & GaSe & HIGH \\
\hline $\mathrm{GaBiSe}_{2}$ & GaSe & HIGH \\
\hline $\mathrm{GaBiTe}_{2}$ & GaSe & HIGH \\
\hline $\mathrm{InNO}_{2}$ & GaSe & MEDIUM \\
\hline $\mathrm{InNS}_{2}$ & GaSe & LOW \\
\hline $\mathrm{InNSe}_{2}$ & GaSe & LOW \\
\hline $\mathrm{InNTe}_{2}$ & GaSe & LOW \\
\hline $\mathrm{InPO}_{2}$ & GaSe & MEDIUM \\
\hline $\mathrm{InPS}_{2}$ & GaSe & $\mathrm{HIGH}$ \\
\hline $\mathrm{InPSe}_{2}$ & GaSe & $\mathrm{HIGH}$ \\
\hline $\mathrm{InPTe}_{2}$ & GaSe & HIGH \\
\hline $\mathrm{InAsO}_{2}$ & GaSe & MEDIUM \\
\hline $\operatorname{InAs} \mathrm{A}_{2}$ & GaSe & $\mathrm{HIGH}$ \\
\hline $\mathrm{InAsSe}_{2}$ & GaSe & HIGH \\
\hline $\mathrm{InAsTe}_{2}$ & GaSe & HIGH \\
\hline
\end{tabular}




\begin{tabular}{|c|c|c|}
\hline $\mathrm{InSbO}_{2}$ & GaSe & MEDIUM \\
\hline $\mathrm{InSbS}_{2}$ & GaSe & HIGH \\
\hline $\mathrm{InSbSe}_{2}$ & GaSe & HIGH \\
\hline $\operatorname{InSbTe}_{2}$ & GaSe & HIGH \\
\hline $\mathrm{InBiO}_{2}$ & GaSe & MEDIUM \\
\hline $\mathrm{InBiS}_{2}$ & GaSe & HIGH \\
\hline $\mathrm{InBiSe}_{2}$ & GaSe & HIGH \\
\hline $\mathrm{InBiTe}_{2}$ & GaSe & HIGH \\
\hline $\mathrm{TlNO}_{2}$ & GaSe & MEDIUM \\
\hline $\mathrm{TlNS}_{2}$ & GaSe & LOW \\
\hline $\mathrm{TlNSe}_{2}$ & GaSe & LOW \\
\hline $\mathrm{TlNTe}_{2}$ & GaSe & LOW \\
\hline $\mathrm{TlPO}_{2}$ & GaSe & HIGH \\
\hline $\mathrm{TlPS}_{2}$ & GaSe & HIGH \\
\hline $\mathrm{TlPSe}_{2}$ & GaSe & HIGH \\
\hline $\mathrm{TlPTe}_{2}$ & GaSe & HIGH \\
\hline $\mathrm{TlAsO}_{2}$ & GaSe & HIGH \\
\hline $\mathrm{TlAsS}_{2}$ & GaSe & HIGH \\
\hline $\mathrm{TlAsSe}_{2}$ & GaSe & HIGH \\
\hline $\mathrm{TlAsTe}_{2}$ & GaSe & HIGH \\
\hline $\mathrm{TlSbO}_{2}$ & GaSe & HIGH \\
\hline $\mathrm{TlSbS}_{2}$ & GaSe & HIGH \\
\hline $\mathrm{TlSbSe}_{2}$ & GaSe & HIGH \\
\hline $\mathrm{TlSbTe}_{2}$ & GaSe & HIGH \\
\hline $\mathrm{TlBiO}_{2}$ & GaSe & HIGH \\
\hline $\mathrm{TlBiS}_{2}$ & GaSe & HIGH \\
\hline $\mathrm{TlBiSe}_{2}$ & GaSe & HIGH \\
\hline
\end{tabular}




$\begin{array}{llc}\mathrm{TlBiTe}_{2} & \text { GaSe } & \text { HIGH } \\ \mathrm{BNO}_{2} & \text { AuSe } & \text { MEDIUM } \\ \mathrm{BNS}_{2} & \text { AuSe } & \text { LOW } \\ \mathrm{BNSe}_{2} & \text { AuSe } & \text { LOW } \\ \mathrm{BNTe}_{2} & \text { AuSe } & \text { MEDIUM } \\ \mathrm{BPO}_{2} & \text { AuSe } & \text { MEDIUM } \\ \mathrm{BPS}_{2} & \text { AuSe } & \text { LOW } \\ \mathrm{BPSe}_{2} & \text { AuSe } & \text { LOW } \\ \mathrm{BPTe}_{2} & \text { AuSe } & \text { LOW } \\ \mathrm{BAsO}_{2} & \text { AuSe } & \text { MEDIUM } \\ \mathrm{BAsS}_{2} & \text { AuSe } & \text { LOW } \\ \mathrm{BAsSe}_{2} & \text { AuSe } & \text { LOW } \\ \mathrm{BAsTe}_{2} & \text { AuSe } & \text { LOW } \\ \mathrm{BSbO}_{2} & \text { AuSe } & \text { MEDIUM } \\ \mathrm{BSbS}_{2} & \text { AuSe } & \text { LOW } \\ \mathrm{BSbSe}_{2} & \text { AuSe } & \text { LOW } \\ \mathrm{BSbTe}_{2} & \text { AuSe } & \text { LOW } \\ \mathrm{BBiO}_{2} & \text { AuSe } & \text { MEDIUM } \\ \mathrm{BBiS}_{2} & \text { AuSe } & \text { LOW } \\ \mathrm{BBiSe}_{2} & \text { AuSe } & \text { LOW } \\ \mathrm{BBiTe}_{2} & \text { AuSe } & \text { LOW } \\ \mathrm{AlNO}_{2} & \text { AuSe } & \text { MEDIUM } \\ \mathrm{AlNS}_{2} & \text { AuSe } & \text { MEDIUM } \\ \mathrm{AlNSe}_{2} & \text { AuSe } & \text { MEDIUM } \\ \mathrm{AlNTe}_{2} & \text { AuSe } & \text { LOW } \\ \mathrm{AlPO}_{2} & \text { AuSe } & \text { MEDIUM } \\ \mathrm{AlPS}_{2} & \text { AuSe } & \text { HIGH }\end{array}$




$\begin{array}{llc}\mathrm{AlPSe}_{2} & \text { AuSe } & \text { HIGH } \\ \mathrm{AlPTe}_{2} & \text { AuSe } & \text { HIGH } \\ \mathrm{AlAsO}_{2} & \text { AuSe } & \text { MEDIUM } \\ \mathrm{AlAsS}_{2} & \text { AuSe } & \text { HIGH } \\ \mathrm{AlAsSe}_{2} & \text { AuSe } & \text { HIGH } \\ \mathrm{AlAsTe}_{2} & \text { AuSe } & \text { HIGH } \\ \mathrm{AlSbO}_{2} & \text { AuSe } & \text { MEDIUM } \\ \mathrm{AlSbS}_{2} & \text { AuSe } & \text { HIGH } \\ \mathrm{AlSbSe}_{2} & \text { AuSe } & \text { HIGH } \\ \mathrm{AlSbTe}_{2} & \text { AuSe } & \text { HIGH } \\ \mathrm{AlBiO}_{2} & \text { AuSe } & \text { MEDIUM } \\ \mathrm{AlBiS}_{2} & \text { AuSe } & \text { HIGH } \\ \mathrm{AlBiSe}_{2} & \text { AuSe } & \text { HIGH } \\ \mathrm{AlBiTe}_{2} & \text { AuSe } & \text { HIGH } \\ \mathrm{GaNO}_{2} & \text { AuSe } & \text { MEDIUM } \\ \mathrm{GaNS}_{2} & \text { AuSe } & \text { MEDIUM } \\ \mathrm{GaNSe}_{2} & \text { AuSe } & \text { MEDIUM } \\ \mathrm{GaNTe}_{2} & \text { AuSe } & \text { LOW } \\ \mathrm{GaPO}_{2} & \text { AuSe } & \text { MEDIUM } \\ \mathrm{GaPS}_{2} & \text { AuSe } & \text { HIGH } \\ \mathrm{GaPSe}_{2} & \text { AuSe } & \text { HIGH } \\ \mathrm{GaPTe}_{2} & \text { AuSe } & \text { HIGH } \\ \mathrm{GaAsO}_{2} & \text { AuSe } & \text { MEDIUM } \\ \mathrm{GaAsS}_{2} & \text { AuSe } & \text { HIGH } \\ \mathrm{GaAsSe}_{2} & \text { AuSe } & \text { HIGH } \\ \mathrm{GaAsTe}_{2} & \text { AuSe } & \text { LOW } \\ \mathrm{GaSbO}_{2} & \text { AuSe } & \text { MEDIUM }\end{array}$




\begin{tabular}{|c|c|c|}
\hline $\mathrm{GaSbS}_{2}$ & $\mathrm{AuSe}$ & $\mathrm{HIGH}$ \\
\hline $\mathrm{GaSbSe}_{2}$ & $\mathrm{AuSe}$ & MEDIUM \\
\hline $\mathrm{GaSbTe}_{2}$ & $\mathrm{AuSe}$ & LOW \\
\hline $\mathrm{GaBiO}_{2}$ & $\mathrm{AuSe}$ & MEDIUM \\
\hline $\mathrm{GaBiS}_{2}$ & $\mathrm{AuSe}$ & HIGH \\
\hline $\mathrm{GaBiSe}_{2}$ & $\mathrm{AuSe}$ & HIGH \\
\hline $\mathrm{GaBiTe}_{2}$ & AuSe & HIGH \\
\hline $\mathrm{InNO}_{2}$ & AuSe & MEDIUM \\
\hline $\mathrm{InNS}_{2}$ & $\mathrm{AuSe}$ & LOW \\
\hline $\mathrm{InNSe}_{2}$ & $\mathrm{AuSe}$ & LOW \\
\hline $\mathrm{InNTe}_{2}$ & $\mathrm{AuSe}$ & LOW \\
\hline $\mathrm{InPO}_{2}$ & $\mathrm{AuSe}$ & MEDIUM \\
\hline $\mathrm{InPS}_{2}$ & $\mathrm{AuSe}$ & HIGH \\
\hline $\mathrm{InPSe}_{2}$ & $\mathrm{AuSe}$ & HIGH \\
\hline $\mathrm{InPTe}_{2}$ & AuSe & HIGH \\
\hline $\mathrm{InAsO}_{2}$ & $\mathrm{AuSe}$ & MEDIUM \\
\hline $\operatorname{InAs} \mathrm{S}_{2}$ & $\mathrm{AuSe}$ & HIGH \\
\hline InAsSe ${ }_{2}$ & $\mathrm{AuSe}$ & MEDIUM \\
\hline $\mathrm{InAsTe}_{2}$ & $\mathrm{AuSe}$ & LOW \\
\hline $\mathrm{InSbO}_{2}$ & AuSe & MEDIUM \\
\hline $\mathrm{InSbS}_{2}$ & $\mathrm{AuSe}$ & MEDIUM \\
\hline $\mathrm{InSbSe}_{2}$ & AuSe & MEDIUM \\
\hline $\mathrm{InSbTe}_{2}$ & $\mathrm{AuSe}$ & HIGH \\
\hline $\mathrm{InBiO}_{2}$ & $\mathrm{AuSe}$ & MEDIUM \\
\hline $\mathrm{InBiS}_{2}$ & $\mathrm{AuSe}$ & HIGH \\
\hline $\mathrm{InBiSe}_{2}$ & $\mathrm{AuSe}$ & HIGH \\
\hline $\mathrm{InBiTe}_{2}$ & $\mathrm{AuSe}$ & HIGH \\
\hline
\end{tabular}




$\begin{array}{llc}\mathrm{TlNO}_{2} & \text { AuSe } & \text { MEDIUM } \\ \mathrm{TlNS}_{2} & \text { AuSe } & \text { LOW } \\ \mathrm{TlNSe}_{2} & \text { AuSe } & \text { LOW } \\ \mathrm{TlNTe}_{2} & \text { AuSe } & \text { LOW } \\ \mathrm{TlPO}_{2} & \text { AuSe } & \text { MEDIUM } \\ \mathrm{TlPS}_{2} & \text { AuSe } & \text { HIGH } \\ \mathrm{TlPSe}_{2} & \text { AuSe } & \text { HIGH } \\ \mathrm{TlPTe}_{2} & \text { AuSe } & \text { HIGH } \\ \mathrm{TlAsO}_{2} & \text { AuSe } & \text { MEDIUM } \\ \mathrm{TlAsS}_{2} & \text { AuSe } & \text { HIGH } \\ \mathrm{TlAsSe}_{2} & \text { AuSe } & \text { HIGH } \\ \mathrm{TlAsTe}_{2} & \text { AuSe } & \text { HIGH } \\ \mathrm{TlSbO}_{2} & \text { AuSe } & \text { MEDIUM } \\ \mathrm{TlSbS}_{2} & \text { AuSe } & \text { HIGH } \\ \mathrm{TlSbSe}_{2} & \text { AuSe } & \text { HIGH } \\ \mathrm{TlSbTe}_{2} & \text { AuSe } & \text { HIGH } \\ \mathrm{TlBiO}_{2} & \text { AuSe } & \text { MEDIUM } \\ \mathrm{TlBiS}_{2} & \text { AuSe } & \text { HIGH } \\ \mathrm{TlBiSe}_{2} & \text { AuSe } & \text { HIGH } \\ \mathrm{TlBiTe}_{2} & \text { AuSe } & \text { HIGH } \\ \mathrm{BNO}_{2} & \text { NiSe } & \text { MEDIUM } \\ \mathrm{BNS}_{2} & \text { NiSe } & \text { LOW } \\ \mathrm{BNSe}_{2} & \text { NiSe } & \text { LOW } \\ \mathrm{BNTe}_{2} & \text { NiSe } & \text { MEDIUM } \\ \mathrm{BPO}_{2} & \text { NiSe } & \text { MEDIUM } \\ \mathrm{BPS}_{2} & \text { NiSe } & \text { LOW } \\ & \text { NiSe } & \text { LOW }\end{array}$




\begin{tabular}{|c|c|c|}
\hline $\mathrm{BPTe}_{2}$ & $\mathrm{NiSe}$ & LOW \\
\hline $\mathrm{BAsO}_{2}$ & NiSe & MEDIUM \\
\hline $\mathrm{BAsS}_{2}$ & $\mathrm{NiSe}$ & LOW \\
\hline $\mathrm{BAsSe}_{2}$ & NiSe & LOW \\
\hline $\mathrm{BAsTe}_{2}$ & $\mathrm{NiSe}$ & LOW \\
\hline $\mathrm{BSbO}_{2}$ & $\mathrm{NiSe}$ & MEDIUM \\
\hline $\mathrm{BSbS}_{2}$ & $\mathrm{NiSe}$ & LOW \\
\hline $\mathrm{BSbSe}_{2}$ & $\mathrm{NiSe}$ & LOW \\
\hline $\mathrm{BSbTe}_{2}$ & NiSe & LOW \\
\hline $\mathrm{BBiO}_{2}$ & NiSe & MEDIUM \\
\hline $\mathrm{BBiS}_{2}$ & NiSe & LOW \\
\hline $\mathrm{BBiSe}_{2}$ & NiSe & LOW \\
\hline $\mathrm{BBiTe}_{2}$ & $\mathrm{NiSe}$ & LOW \\
\hline $\mathrm{AlNO}_{2}$ & NiSe & MEDIUM \\
\hline $\mathrm{AlNS}_{2}$ & NiSe & MEDIUM \\
\hline $\mathrm{AlNSe}_{2}$ & NiSe & MEDIUM \\
\hline $\mathrm{AlNTe}_{2}$ & NiSe & MEDIUM \\
\hline $\mathrm{AlPO}_{2}$ & $\mathrm{NiSe}$ & MEDIUM \\
\hline $\mathrm{AlPS}_{2}$ & $\mathrm{NiSe}$ & $\mathrm{HIGH}$ \\
\hline $\mathrm{AlPSe}_{2}$ & $\mathrm{NiSe}$ & HIGH \\
\hline $\mathrm{AlPTe}_{2}$ & $\mathrm{NiSe}$ & $\mathrm{HIGH}$ \\
\hline $\mathrm{AlAsO}_{2}$ & NiSe & MEDIUM \\
\hline $\mathrm{AlAsS}_{2}$ & NiSe & HIGH \\
\hline $\mathrm{AlAsSe}_{2}$ & NiSe & HIGH \\
\hline $\mathrm{AlAsTe}_{2}$ & $\mathrm{NiSe}$ & $\mathrm{HIGH}$ \\
\hline $\mathrm{AlSbO}_{2}$ & NiSe & MEDIUM \\
\hline $\mathrm{AlSbS}_{2}$ & NiSe & HIGH \\
\hline
\end{tabular}




\begin{tabular}{|c|c|c|}
\hline $\mathrm{AlSbSe}_{2}$ & $\mathrm{NiSe}$ & HIGH \\
\hline $\mathrm{AlSbTe}_{2}$ & NiSe & HIGH \\
\hline $\mathrm{AlBiO}_{2}$ & NiSe & MEDIUM \\
\hline $\mathrm{AlBiS}_{2}$ & $\mathrm{NiSe}$ & HIGH \\
\hline $\mathrm{AlBiSe}_{2}$ & $\mathrm{NiSe}$ & HIGH \\
\hline $\mathrm{AlBiTe}_{2}$ & $\mathrm{NiSe}$ & HIGH \\
\hline $\mathrm{GaNO}_{2}$ & $\mathrm{NiSe}$ & MEDIUM \\
\hline $\mathrm{GaNS}_{2}$ & $\mathrm{NiSe}$ & MEDIUM \\
\hline $\mathrm{GaNSe}_{2}$ & NiSe & MEDIUM \\
\hline $\mathrm{GaNTe}_{2}$ & NiSe & LOW \\
\hline $\mathrm{GaPO}_{2}$ & $\mathrm{NiSe}$ & MEDIUM \\
\hline $\mathrm{GaPS}_{2}$ & NiSe & HIGH \\
\hline $\mathrm{GaPSe}_{2}$ & NiSe & HIGH \\
\hline $\mathrm{GaPTe}_{2}$ & $\mathrm{NiSe}$ & HIGH \\
\hline $\mathrm{GaAsO}_{2}$ & NiSe & MEDIUM \\
\hline $\mathrm{GaAsS}_{2}$ & NiSe & HIGH \\
\hline $\mathrm{GaAsSe}_{2}$ & NiSe & HIGH \\
\hline $\mathrm{GaAsTe}_{2}$ & $\mathrm{NiSe}$ & HIGH \\
\hline $\mathrm{GaSbO}_{2}$ & $\mathrm{NiSe}$ & MEDIUM \\
\hline $\mathrm{GaSbS}_{2}$ & $\mathrm{NiSe}$ & HIGH \\
\hline $\mathrm{GaSbSe}_{2}$ & $\mathrm{NiSe}$ & HIGH \\
\hline $\mathrm{GaSbTe}_{2}$ & NiSe & HIGH \\
\hline $\mathrm{GaBiO}_{2}$ & $\mathrm{NiSe}$ & MEDIUM \\
\hline $\mathrm{GaBiS}_{2}$ & NiSe & HIGH \\
\hline $\mathrm{GaBiSe}_{2}$ & $\mathrm{NiSe}$ & HIGH \\
\hline $\mathrm{GaBiTe}_{2}$ & $\mathrm{NiSe}$ & HIGH \\
\hline $\mathrm{InNO}_{2}$ & NiSe & MEDIUM \\
\hline
\end{tabular}




\begin{tabular}{|c|c|c|}
\hline $\mathrm{InNS}_{2}$ & NiSe & LOW \\
\hline $\mathrm{InNSe}_{2}$ & NiSe & LOW \\
\hline $\operatorname{InNTe}_{2}$ & NiSe & LOW \\
\hline $\mathrm{InPO}_{2}$ & NiSe & MEDIUM \\
\hline $\mathrm{InPS}_{2}$ & $\mathrm{NiSe}$ & HIGH \\
\hline $\mathrm{InPSe}_{2}$ & $\mathrm{NiSe}$ & HIGH \\
\hline $\mathrm{InPTe}_{2}$ & $\mathrm{NiSe}$ & HIGH \\
\hline $\mathrm{InAsO}_{2}$ & $\mathrm{NiSe}$ & MEDIUM \\
\hline $\operatorname{In} \mathrm{AsS}_{2}$ & NiSe & HIGH \\
\hline InAsSe ${ }_{2}$ & NiSe & HIGH \\
\hline $\operatorname{InAsTe}_{2}$ & NiSe & HIGH \\
\hline $\mathrm{InSbO}_{2}$ & NiSe & MEDIUM \\
\hline $\mathrm{InSbS}_{2}$ & $\mathrm{NiSe}$ & MEDIUM \\
\hline $\mathrm{InSbSe}_{2}$ & $\mathrm{NiSe}$ & HIGH \\
\hline $\mathrm{InSbTe}_{2}$ & NiSe & HIGH \\
\hline $\mathrm{InBiO}_{2}$ & NiSe & MEDIUM \\
\hline $\mathrm{InBiS}_{2}$ & NiSe & HIGH \\
\hline $\mathrm{InBiSe}_{2}$ & $\mathrm{NiSe}$ & HIGH \\
\hline $\mathrm{InBiTe}_{2}$ & NiSe & HIGH \\
\hline $\mathrm{TlNO}_{2}$ & $\mathrm{NiSe}$ & MEDIUM \\
\hline $\mathrm{TlNS}_{2}$ & $\mathrm{NiSe}$ & LOW \\
\hline $\mathrm{TlNSe}_{2}$ & NiSe & LOW \\
\hline $\mathrm{TlNTe}_{2}$ & NiSe & LOW \\
\hline $\mathrm{TlPO}_{2}$ & NiSe & MEDIUM \\
\hline $\mathrm{TlPS}_{2}$ & $\mathrm{NiSe}$ & HIGH \\
\hline $\mathrm{TlPSe}_{2}$ & $\mathrm{NiSe}$ & HIGH \\
\hline $\mathrm{TlPTe}_{2}$ & NiSe & HIGH \\
\hline
\end{tabular}




\begin{tabular}{|c|c|c|}
\hline $\mathrm{TlAsO}_{2}$ & $\mathrm{NiSe}$ & MEDIUM \\
\hline $\mathrm{TlAsS}_{2}$ & NiSe & HIGH \\
\hline $\mathrm{TlAsSe}_{2}$ & $\mathrm{NiSe}$ & HIGH \\
\hline $\mathrm{TlAsTe}_{2}$ & NiSe & HIGH \\
\hline $\mathrm{TlSbO}_{2}$ & NiSe & MEDIUM \\
\hline $\mathrm{TlSbS}_{2}$ & NiSe & $\mathrm{HIGH}$ \\
\hline $\mathrm{TlSbSe}_{2}$ & $\mathrm{NiSe}$ & HIGH \\
\hline $\mathrm{TlSbTe}_{2}$ & $\mathrm{NiSe}$ & HIGH \\
\hline $\mathrm{TlBiO}_{2}$ & $\mathrm{NiSe}$ & HIGH \\
\hline $\mathrm{TlBiS}_{2}$ & NiSe & HIGH \\
\hline $\mathrm{TlBiSe}_{2}$ & NiSe & HIGH \\
\hline $\mathrm{TlBiTe}_{2}$ & NiSe & HIGH \\
\hline $\mathrm{BNO}_{2}$ & $\mathrm{FeSe}$ & MEDIUM \\
\hline $\mathrm{BNS}_{2}$ & FeSe & LOW \\
\hline $\mathrm{BNSe}_{2}$ & $\mathrm{FeSe}$ & LOW \\
\hline $\mathrm{BNTe}_{2}$ & FeSe & LOW \\
\hline $\mathrm{BPO}_{2}$ & $\mathrm{FeSe}$ & MEDIUM \\
\hline $\mathrm{BPS}_{2}$ & $\mathrm{FeSe}$ & LOW \\
\hline $\mathrm{BPSe}_{2}$ & $\mathrm{FeSe}$ & LOW \\
\hline $\mathrm{BPTe}_{2}$ & $\mathrm{FeSe}$ & LOW \\
\hline $\mathrm{BAsO}_{2}$ & $\mathrm{FeSe}$ & MEDIUM \\
\hline $\mathrm{BAsS}_{2}$ & $\mathrm{FeSe}$ & LOW \\
\hline $\mathrm{BAsSe}_{2}$ & $\mathrm{FeSe}$ & LOW \\
\hline $\mathrm{BAsTe}_{2}$ & FeSe & LOW \\
\hline $\mathrm{BSbO}_{2}$ & $\mathrm{FeSe}$ & MEDIUM \\
\hline $\mathrm{BSbS}_{2}$ & $\mathrm{FeSe}$ & LOW \\
\hline $\mathrm{BSbSe}_{2}$ & FeSe & LOW \\
\hline
\end{tabular}




$\begin{array}{llc}\mathrm{BSbTe}_{2} & \text { FeSe } & \text { LOW } \\ \mathrm{BBiO}_{2} & \text { FeSe } & \text { MEDIUM } \\ \mathrm{BBiS}_{2} & \text { FeSe } & \text { LOW } \\ \mathrm{BBiSe}_{2} & \text { FeSe } & \text { LOW } \\ \mathrm{BBiTe}_{2} & \text { FeSe } & \text { LOW } \\ \mathrm{AlNO}_{2} & \text { FeSe } & \text { MEDIUM } \\ \mathrm{AlNS}_{2} & \text { FeSe } & \text { MEDIUM } \\ \mathrm{AlNSe}_{2} & \text { FeSe } & \text { MEDIUM } \\ \mathrm{AlNTe}_{2} & \text { FeSe } & \text { LOW } \\ \mathrm{AlPO}_{2} & \text { FeSe } & \text { MEDIUM } \\ \mathrm{AlPS}_{2} & \text { FeSe } & \text { HIGH } \\ \mathrm{AlPSe}_{2} & \text { FeSe } & \text { HIGH } \\ \mathrm{AlPTe}_{2} & \text { FeSe } & \text { HIGH } \\ \mathrm{AlAsO}_{2} & \text { FeSe } & \text { MEDIUM } \\ \mathrm{AlAsS}_{2} & \text { FeSe } & \text { HIGH } \\ \mathrm{AlAsSe}_{2} & \text { FeSe } & \text { HIGH } \\ \mathrm{AlAsTe}_{2} & \text { FeSe } & \text { HIGH } \\ \mathrm{AlSbO}_{2} & \text { FeSe } & \text { MEDIUM } \\ \mathrm{AlSbS}_{2} & \text { FeSe } & \text { HIGH } \\ \mathrm{AlSbSe}_{2} & \text { FeSe } & \text { HIGH } \\ \mathrm{AlSbTe}_{2} & \text { FeSe } & \text { HIGH } \\ \mathrm{AlBiO}_{2} & \text { FeSe } & \text { MEDIUM } \\ \mathrm{AlBiS}_{2} & \text { FeSe } & \text { HIGH } \\ \mathrm{AlBiSe}_{2} & \text { FeSe } & \text { HIGH } \\ \mathrm{AlBiTe}_{2} & \text { FeSe } & \text { HIGH } \\ \mathrm{GaNO}_{2} & \text { FeSe } & \text { HIGH } \\ & \text { FeSe } & \text { MEDIUM }\end{array}$




$\begin{array}{llc}\mathrm{GaNSe}_{2} & \text { FeSe } & \text { LOW } \\ \mathrm{GaNTe}_{2} & \text { FeSe } & \text { LOW } \\ \mathrm{GaPO}_{2} & \text { FeSe } & \text { HIGH } \\ \mathrm{GaPS}_{2} & \text { FeSe } & \text { HIGH } \\ \mathrm{GaPSe}_{2} & \text { FeSe } & \text { HIGH } \\ \mathrm{GaPTe}_{2} & \text { FeSe } & \text { HIGH } \\ \mathrm{GaAsO}_{2} & \text { FeSe } & \text { HIGH } \\ \mathrm{GaAsS}_{2} & \text { FeSe } & \text { HIGH } \\ \mathrm{GaAsSe}_{2} & \text { FeSe } & \text { HIGH } \\ \mathrm{GaAsTe}_{2} & \text { FeSe } & \text { HIGH } \\ \mathrm{GaSbO}_{2} & \text { FeSe } & \text { MEDIUM } \\ \mathrm{GaSbS}_{2} & \text { FeSe } & \text { HIGH } \\ \mathrm{GaSbSe}_{2} & \text { FeSe } & \text { HIGH } \\ \mathrm{GaSbTe}_{2} & \text { FeSe } & \text { HIGH } \\ \mathrm{GaBiO}_{2} & \text { FeSe } & \text { MEDIUM } \\ \mathrm{GaBiS}_{2} & \text { FeSe } & \text { HIGH } \\ \mathrm{GaBiSe}_{2} & \text { FeSe } & \text { HIGH } \\ \mathrm{GaBiTe}_{2} & \text { FeSe } & \text { HIGH } \\ \mathrm{InNO}_{2} & \text { FeSe } & \text { MEDIUM } \\ \mathrm{InNTS}_{2} & \text { FeSe } & \text { LOW } \\ \mathrm{InNSe}_{2} & \text { FeSe } & \text { LOW } \\ \mathrm{InNTe}_{2} & \text { FeSe } & \text { LOW } \\ \mathrm{InPO}_{2} & \text { FeSe } & \text { MEDIUM } \\ \text { InPSe }_{2} & \text { FeSe } & \text { HIGH } \\ \text { FeSe } & \text { HIGH } \\ \text { HIGH } \\ \text { FEDIUM }\end{array}$




\begin{tabular}{|c|c|c|}
\hline $\mathrm{InAsS}_{2}$ & FeSe & HIGH \\
\hline $\operatorname{InAsSe}{ }_{2}$ & $\mathrm{FeSe}$ & HIGH \\
\hline $\mathrm{InAsTe}_{2}$ & $\mathrm{FeSe}$ & HIGH \\
\hline $\mathrm{InSbO}_{2}$ & $\mathrm{FeSe}$ & MEDIUM \\
\hline $\mathrm{InSbS}_{2}$ & $\mathrm{FeSe}$ & HIGH \\
\hline $\mathrm{InSbSe}_{2}$ & $\mathrm{FeSe}$ & HIGH \\
\hline $\mathrm{InSbTe}_{2}$ & $\mathrm{FeSe}$ & HIGH \\
\hline $\mathrm{InBiO}_{2}$ & $\mathrm{FeSe}$ & HIGH \\
\hline $\mathrm{InBiS}_{2}$ & $\mathrm{FeSe}$ & HIGH \\
\hline $\mathrm{InBiSe}_{2}$ & $\mathrm{FeSe}$ & HIGH \\
\hline $\mathrm{InBiTe}_{2}$ & $\mathrm{FeSe}$ & HIGH \\
\hline $\mathrm{TlNO}_{2}$ & FeSe & MEDIUM \\
\hline $\mathrm{TINS}_{2}$ & $\mathrm{FeSe}$ & LOW \\
\hline $\mathrm{TlNSe}_{2}$ & $\mathrm{FeSe}$ & LOW \\
\hline $\mathrm{TlNTe}_{2}$ & $\mathrm{FeSe}$ & LOW \\
\hline $\mathrm{TlPO}_{2}$ & $\mathrm{FeSe}$ & HIGH \\
\hline $\mathrm{TlPS}_{2}$ & $\mathrm{FeSe}$ & HIGH \\
\hline $\mathrm{TlPSe}_{2}$ & $\mathrm{FeSe}$ & HIGH \\
\hline $\mathrm{TlPTe}_{2}$ & $\mathrm{FeSe}$ & HIGH \\
\hline $\mathrm{TlAsO}_{2}$ & $\mathrm{FeSe}$ & $\mathrm{HIGH}$ \\
\hline $\mathrm{TlAsS}_{2}$ & FeSe & $\mathrm{HIGH}$ \\
\hline $\mathrm{TlAsSe}_{2}$ & $\mathrm{FeSe}$ & HIGH \\
\hline $\mathrm{TlAsTe}_{2}$ & $\mathrm{FeSe}$ & HIGH \\
\hline $\mathrm{TlSbO}_{2}$ & $\mathrm{FeSe}$ & HIGH \\
\hline $\mathrm{TlSbS}_{2}$ & FeSe & HIGH \\
\hline $\mathrm{TlSbSe}_{2}$ & $\mathrm{FeSe}$ & $\mathrm{HIGH}$ \\
\hline $\mathrm{TlSbTe}_{2}$ & FeSe & HIGH \\
\hline
\end{tabular}




$\begin{array}{lll}\mathrm{TlBiO}_{2} & \text { FeSe } & \text { HIGH } \\ \text { TlBiS }_{2} & \text { FeSe } & \text { HIGH } \\ \text { TlBiSe }_{2} & \text { FeSe } & \text { HIGH } \\ \text { TlBiTe }_{2} & \text { FeSe } & \text { HIGH }\end{array}$

